\title{
Synthetic antigen-presenting cells reveal the diversity and functional specialisation of extracellular vesicles composing the fourth signal of $\mathbf{T}$ cell immunological synapses.
}

Pablo F. Céspedes ${ }^{1,11, *}$, Ashwin Jainarayanan ${ }^{1,11}$, Lola Fernández-Messina, ${ }^{2,3}$, David G. Saliba ${ }^{1}$, Salvatore Valvo ${ }^{1}$, Audun Kvalvaag ${ }^{1}$, Lina $\mathrm{Chen}^{1}$, Elke Kurz ${ }^{1}$, Charity Ganskow ${ }^{1}$, Huw Colin-York ${ }^{1,4}$, Marco Fritzsche ${ }^{1,4}$, Yanchun Peng ${ }^{4,5}$, Tao Dong ${ }^{4,5}$, Errin Johnson ${ }^{6}$, Jesús A. Siller-Farfán ${ }^{6}$, Omer Dushek ${ }^{6}$, Erdinc Sezgin ${ }^{7}$, Ben Peacock ${ }^{8}$, Alice Law ${ }^{8}$, Dimitri Aubert $^{8}$, Simon Engledow ${ }^{9}$, Moustafa Attar ${ }^{1,9}$, Svenja Hester ${ }^{10}$, Roman Fischer $^{10}$, Francisco Sánchez-Madrid ${ }^{2,3}$, Michael L. Dustin ${ }^{1, *}$.

\section{Affiliations:}

1. Kennedy Institute of Rheumatology, Nuffield Department of Orthopedics, Rheumatology and Musculoskeletal Sciences, The University of Oxford, Oxford, UK.

2. Immunology Service, Hospital de la Princesa, Instituto Investigación Sanitaria Princesa, Universidad Autónoma de Madrid, Madrid, Spain.

3. Intercellular communication in the inflammatory response. Vascular Physiology Area, Centro Nacional de Investigaciones Cardiovasculares (CNIC), Madrid, Spain.

4. MRC Human Immunology Unit, MRC Weatherall Institute of Molecular Medicine, Radcliffe Department of Medicine, The University of Oxford, Oxford, UK.

5. Chinese Academy of Medical Science (CAMS) Oxford Institute (COI), University of Oxford, Oxford, UK.

6. Sir William Dunn School of Pathology, The University of Oxford, Oxford, UK.

7. Science for Life Laboratory, Department of Women's and Children's Health, Karolinska Institutet, Stockholm, Sweden.

8. NanoFCM, MediCity, Nottingham, UK.

9. Oxford Genomics Centre, Wellcome Centre for Human Genetics, The University of Oxford, Oxford, UK.

10. Target Discovery Institute, Centre for Medicines Discovery, Nuffield Department of Medicine, The University of Oxford, Oxford, UK.

11. These authors contributed equally to this work.

*. To whom any correspondence should be addressed. 


\section{Graphical abstract}

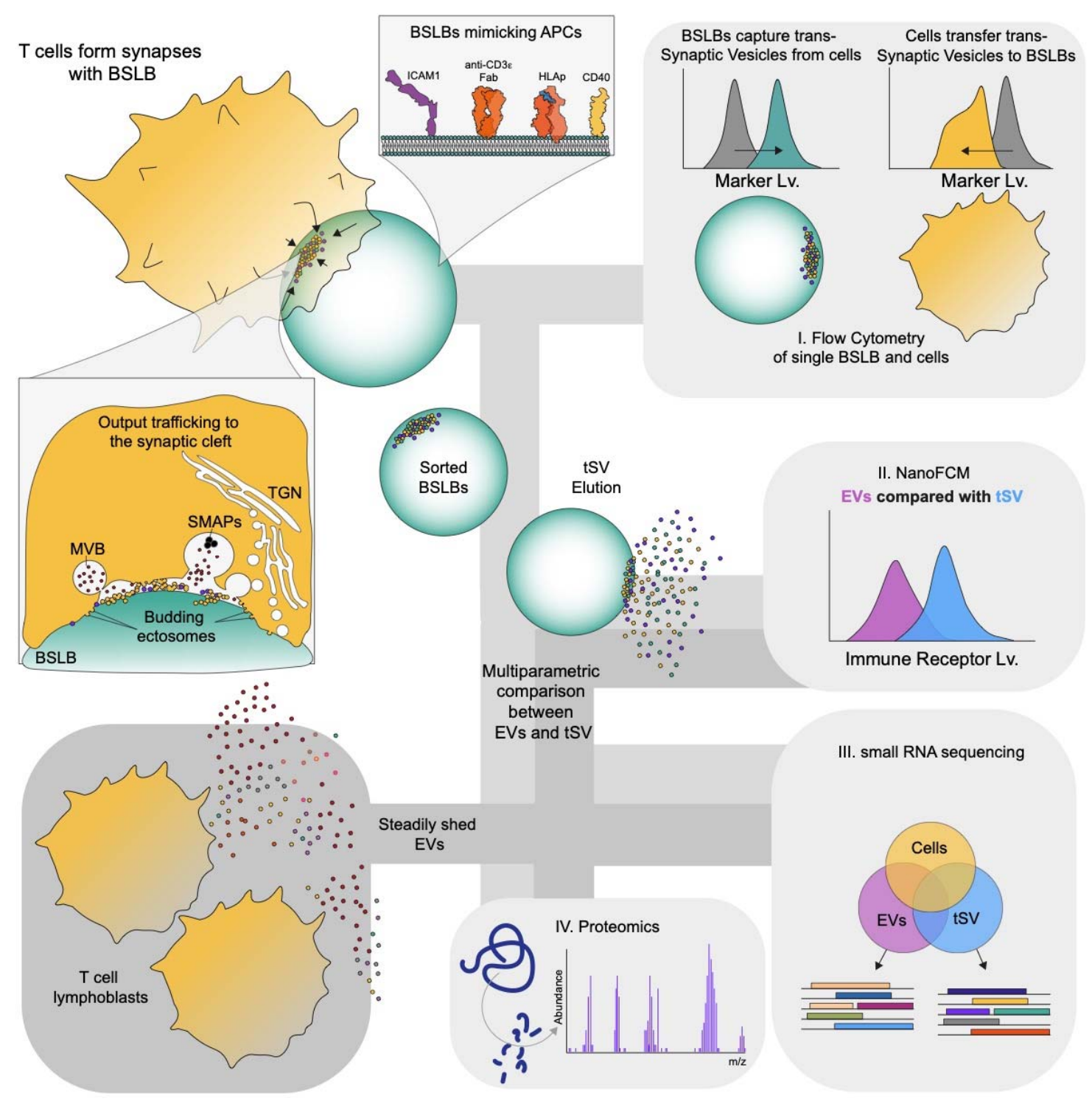

\section{Highlights}

- Bead Supported Lipid Bilayers (BSLB) reconstituting antigen-presenting cells support synapse assembly by $\mathrm{T}$ cells and the release of effector particles.

- BSLB facilitate the dissection of the cellular machineries and synapse composition shaping the released $\mathrm{tSV}$.

- $\quad$ tSV and their steadily released counterparts have a different composition. TSV show a higher enrichment of effectors including immune receptors, miR, RNA- and other nucleic acid-binding proteins, than EVs. 


\section{ABSTRACT}

The T cell Immunological Synapse (IS) is a pivotal hub for the regulation of adaptive immunity by endowing the exchange of information between cells engaged in physical contacts. Beyond the integration of antigen (signal one), co-stimulation (signal two), and cytokines (signal three), the IS facilitates the delivery of T-cell effector assemblies including supramolecular attack particles (SMAPs) and extracellular vesicles (EVs). How these particulate outputs differ among $\mathrm{T}$-cell subsets and how subcellular compartments and signals exchanged at the synapse contribute to their composition is not fully understood. Here we harnessed bead-supported lipid bilayers (BSLBs) as a tailorable and versatile technology for the study of synaptic particle biogenesis and composition in different T-cell subsets, including CART. These synthetic antigen-presenting cells (APCs) facilitated the characterisation of trans-synaptic vesicles (tSV) as a heterogeneous population of EVs comprising among others PM-derived synaptic ectosomes and $\mathrm{CD}^{+} 3^{+}$exosomes. We harnessed BSLB to unveil the factors influencing the vesicular release of CD40L, as a model effector, identifying CD40 trans presentation, T-cell activation, ESCRT upregulation/recruitment, antigen density/potency, co-repression by PD-1 ligands, and its processing by ADAM10 as major determinants. Further, BSLB made possible the comparison of microRNA (miR) species associated with tSV and steadily released EVs. Altogether, our data provide evidence for a higher specialisation of tSV which are enriched not only in effector immune receptors but also in miR and RNA-binding proteins. Considering the molecular uniqueness and functional complexity of the tSV output, which is also accompanied by SMAPs, we propose their classification as signal four.

Keywords: $\mathrm{T}$ cells, extracellular vesicles, regulatory $\mathrm{T}$ cells, cytotoxic $\mathrm{T}$ cells, chimeric antigen receptor-expressing $\mathrm{T}$ cells (CART), synaptic ectosomes, trans-synaptic vesicles, 
bioRxiv preprint doi: https://doi.org/10.1101/2021.05.29.445691; this version posted August 19, 2021. The copyright holder for this preprint (which was not certified by peer review) is the author/funder. All rights reserved. No reuse allowed without permission.

CD40L, miRNA, CD38, CD39, CD73, CD80, CD86, PD-L1, PD-L2, HIV gp120, ADAM10, BST2, and CD81. 


\section{INTRODUCTION}

$\mathrm{T}$ lymphocytes are key players in the regulation and promotion of adaptive immunity. Helper T cells (TH), cytotoxic T lymphocytes (CTL) and regulatory $\mathrm{T}$ cells (Treg) shape cellular networks through the assembly of cell-cell interfaces termed Immunological Synapses (IS) with antigen-presenting cells (APCs) and other T cells[1, 2]. These ISs enable three key receptor-ligand driven signals critical to mounting an immune response or maintaining self-tolerance: 1) antigen recognition, 2) co-stimulation and co-repression, and 3) sensing of soluble cytokines released into the synaptic cleft. Here we propose a fourth type of trans-synaptic signal based on trans-synaptic supramolecular effectors and provide a general tool to study these challenging to isolate signaling entities.

In the past 10 years, the IS has been recognized as a site for bidirectional relay of supramolecular effectors, including polarized exosomes (PE) and supramolecular attack particles (SMAPs) released from multivesicular bodies[3] into the synaptic cleft, synaptic ectosome (SE) budding from the T cell plasma membrane (PM) across the synaptic cleft and trans-endocytosis of PM fragments across the synaptic cleft [4-6]. PE and SE are actively formed by the donor $\mathrm{T}$ cells through action of the endosomal sorting complexes for transport (ESCRT) machinery and together make up the trans-synaptic vesicles (tSV). Transendocytosis and trogocytosis result in taking of small or large, respectively, membrane fragments from one cell into another [7-11]. SMAPs are similar in size to exosomes and SE but lack a phospholipid membrane and instead have a core-shell structure that enables transfer of complex cargo[6]. tSVs and SMAPs have been studies by capture on synthetic APC, but the ability to manipulate them after capture has been limited and we offer a new tool through this resource.

While tSV, trans-endocytosed fragments and SMAPs are consumed by the synaptic partner, extracellular vesicles (EVs) formed by the ESCRT machinery and steadily released 
into the media can be collected from the culture supernatant of activated T cells[12]. Previous work has used EVs as a surrogate for tSV. While this approach has had some predictive power, a comparison of $\mathrm{tSV}$ and EVs is critical for progress in the field of cell-cell communication. Here, we develop a resource to collect tSV released from three major types of T cells and compared tSV and sEV released from the same cells. The approach should be applicable to study tSV generated in other settings.

\section{RESULTS}

\section{Trans-synaptic vesicles are larger and carry more cargo than sEV.}

Supported lipid bilayer (SLB) on glass substrates have been used extensively to study cell recognition processes between live cells and SLB presenting molecular components from a natural interaction partner. SLB on planar supports presenting surrogate antigen, adhesion and co-stimulatory receptors were first used to characterize tSV and SMAPs from helper T cells and cytotoxic T cells, respectively. The advantage of the SLB is that following T cell release of tSV or SMAPs, the respective T cells can be selectively released, leaving the intact tSV or SMAPs behind for analysis by imaging or mass spectrometry. SLB can be readily formed on $5.0 \pm 0.05 \mu \mathrm{m}$ glass beads (BSLB) to improve compositional analysis [5]. We have further improved on this first generation of BSLB to develop a general resource for study of tSV and SMAPs in any synaptic model.

Our second generation BSLB model uses a surrogate antigen, a 14 His tagged $\alpha$-CD3 $\varepsilon$ Fab, that can be gently eluted from the SLB to enable release of tSV. We used fluorescence correlation spectroscopy to demonstrate that the recombinant $\alpha-\mathrm{CD} 3 \varepsilon$ Fab and a coincorporated Abberior Star-Red phosphoethanolamine had diffusion coefficients of $0.68 \pm$ 0.39 and $3.2 \pm 0.79 \mu \mathrm{m}^{2} / \mathrm{s}$, respectively, which are comparable to ranges reported for PM proteins and lipids [13-15]. To analyze tSV from helper T cells we used BSLB presenting a 
range of $\alpha-C D 3 \varepsilon$ Fab densities, and physiological densities of the adhesion molecule ICAM1 $\left(200 \mathrm{molec} . / \mu^{2}\right)$ and costimulator receptor CD40 $\left(20 \mathrm{molec} . / \mu \mathrm{m}^{2}\right)$, which are characteristic of natural APCs. Time-lapse imaging of T cell-BSLB co-cultures demonstrated that BSLB instigated transfer of CD40L from stimulated T cells, which left a "synaptic stamp" on the engaged BSLBs composed of the transferred tSV (Fig. 1A). To enable the synchronous release of BSLBs from conjugates, we gradually cooled the cell-BSLB co-culture to $4^{\circ} \mathrm{C}$ to promote dissociation of the LFA1-ICAM1 interaction while minimizing mechanical stress on BSLB and their tethered tSV. Following this treatment, flow cytometry (FCM) revealed the conversion of conjugates to single T cells and BSLB with evidence of $\mathrm{T}$ cell receptor (TCR) downregulation on $\mathrm{T}$ cells and TCR acquisition by BSLB as a function of $\alpha-C D 3 \varepsilon$ Fab density (Fig. 1B and Fig. S1A), as previously reported[5]. While BSLB acquired little CD2 and CD4, T cells acquired little fluorescent lipids (DOPE) indicating limited trogocytosis or trans-endocytosis of material from BSLB (Figs. 1B and S1B), contrasting to with has previously been reported for B cell IS [16].

We next eluted the tSV with ice-cold EDTA from BSLB previously enriched by low speed centrifugation $(100 \mathrm{~g} 1 \mathrm{~min})$ and used differential and ultracentrifugation to concentrate the eluted tSV (see Methods). We focused our analyses on tSV containing the T cell effector molecules TCR and CD40L, the broad EV and PM marker $\mathrm{CD}^{+} 1^{+}$and on $\mathrm{CD}^{+} 3^{+} \mathrm{tSV}$ belonging to exosomes. Most of the TCR, CD40L and CD81 present on BSLBs was eluted under these conditions (Fig. 1C and D). Elution of $\mathrm{CD}^{+} 3^{+}$vesicles was less efficient but still significant (Fig. 1D). Electron microscopy images of eluted tSV revealed classical EV-like profiles (Fig. 1E, arrows). We were now able to compare tSV with steadily released EVs isolated from activated TH cells of the same donors cultured in EV-free media. TSV were larger than EVs by nanoparticle tracking analyses (NTA) (Fig. S1C). This was confirmed by nanoFCM revealing tSV having a median diameter of $82.13 \pm 0.75 \mathrm{~nm}$ to $84.4 \pm 5.99 \mathrm{~nm}$, 
with or without CD40 in BSLB, respectively, compared to EVs from T cells of the same donors with a median diameter of $65 \pm 25 \mathrm{~nm}$. More detailed analyses using silica bead standards for size segmentation revealed a higher frequency of events larger than 113 and $155 \mathrm{~nm}$ in tSV compared with EVs (Fig. S1D). EVs showed a higher frequency of events in the $68 \mathrm{~nm}$ size bin (Fig. S1D). Comparison of marker-specific vesicles within tSV and EVs revealed that $\mathrm{CD} 81^{+} \mathrm{tSV}$ coexisted with $\mathrm{TCR} \alpha \beta^{+}$at higher frequencies in tSV than EVs (Fig. 1F) and a larger median size for $\mathrm{TCR}^{+} \mathrm{tSV}$ than $\mathrm{TCR}^{+} \mathrm{EVs}$ (Fig. S1E,F), respectively. We also observed a larger median size for $\mathrm{CD}_{40 \mathrm{~L}^{+}}$or $\mathrm{CD} 81^{+} \mathrm{tSV}$ than $\mathrm{CD} 40 \mathrm{~L}^{+}$or $\mathrm{CD} 81^{+} \mathrm{EVs}$ (Fig. S1E), whereas no significant size differences were observed for $\mathrm{BST}_{2}^{+}$or $\mathrm{CD}^{+} 3^{+} \mathrm{tSV}$ and EVs. TSV also expressed higher levels of TCR or CD40L than EVs (Fig. 1G,H, and Fig. $\mathrm{S} 1 \mathrm{G}, \mathrm{H})$. Since CD40L detection by antibodies is partly impaired by the presence of CD40 (see also Fig. 3B), we confirmed CD40L elution and compared its expression levels in tSV and EVs by immunoblotting (Fig. $1 \mathrm{H}$ and $\mathrm{S} 1 \mathrm{H}$ ). Most likely, the overall larger size and immune receptor content of $\mathrm{tSV}$ derives from the subpopulation of SE, which contains high densities of TCR and CD40L as microclusters $>80 \mathrm{~nm}[5]$. The membrane-anchored fulllength CD40L was predominantly found $(29.2 \mathrm{kDa})$ in the eluates of BSLBs, with neligible detection of its soluble ectodomain (Fig. S1H). Null CD40 ${ }^{+}$BSLB capture neligible amounts of CD40L from TCR-unstimulated cells, which might relate to the CD40-dependent capture of a fraction of EVs released in response to adhesion (i.e., ICAM1:LFA-1 interaction). The imaging of tSV markers with TIRFM and confocal imaging further revealed that beyond differences in size and immune receptor content, tSV displayed different degrees of colocalisation in intact, non-permeabilised ISs (Fig. S2A-I) reflecting their different subcellular origin and the compositional and spatial heterogeneity of tSV in the synaptic cleft.

TSV are likely composed of TCR $\alpha \beta^{+}, \mathrm{CD} 40 \mathrm{~L}^{+} \mathrm{SE}$ and $\mathrm{CD}^{+} 3^{+} \mathrm{PE}$, which may have different release characteristics. We used the BSLB system to investigate requirements for 
tSV release using a panel of pharmacological inhibitors of candidate steps. A panel of 12 inhibitors targeting cytoskeleton dynamics and the transport of vesicular cargo from different organelles, such as multivesicular bodies (MVB) and the trans-Golgi network (TGN) was used (Table S1 and Fig. S3). Folling the treatment for $90 \mathrm{~min}$ at $37^{\circ} \mathrm{C}$ and $5 \% \mathrm{CO}_{2}$, we monitored TCR, CD40L, BST2, and CD63 transfer from T cells to BSLB (Fig. S3A) and normalised trans-synaptic transfer to the maximum observed in untreated controls $\left(\% \mathrm{~T}_{\max }\right)$.

Actin cytoskeleton disruptors Latrunculin A and Jasplakinolide, but not Cytochalasin D, impaired cell-BSLB conjugates (Fig. S3B). Cytochalasin D and the other inhibirors didn't impair cell-BSLB conjugate formation and thus allowed interrogation of distinct mechanistic steps in delivery of tSV cargo. Fortuitously, we observed a differential inhibition in the transfer of $\mathrm{TCR}^{+}, \mathrm{CD} 4 \mathrm{~L}^{+}, \mathrm{BST}^{+}$and $\mathrm{CD}^{+}{ }^{+} \mathrm{tSV}$ from $\mathrm{TH}$ cells to BSLB, suggesting distinct mechansims of cargo delivery. The transfer of $\mathrm{TCR}^{+} \mathrm{tSV}$ was selectively promoted by dynamin inhibition by Dynasore, and conversely, strongly reduced by inhibition of actin polymerization, ESCRT machinery, and neutral sphingomyelinases (Fig. S3C and Table S1). Reduced endocytosis or increased ubiquitination of TCR following Dynasore treatment [17] might contribute to the observed increase in $\mathrm{TCR}^{+} \mathrm{tSV}$ transfer. The transfer of $\mathrm{CD} 40 \mathrm{~L}^{+} \mathrm{tSV}$ was strongly reduced following acute inhibition of several machineries in the hierarchy TGN $>$ actin dynamics and ESCRT > ceramide synthesis > dynamin > vacuolar $\mathrm{H}^{+}$-ATPases, and class I and II PI3K (Fig S3D). Other members of the TNF superfamily, such as FasL, have also been reported to be affected by interference with TGN trafficking [18, 19], suggesting a conserved mechanism of TNFSF delivery to tSV. In contrast, the transfer of $\mathrm{BST}^{+}$and $\mathrm{CD}^{+} 3^{+} \mathrm{tSV}$ was affected by inhibiting endosomal and lysosomal transport but not by inhibiting TGN transport (Fig. S3E-F). CD40L > TCR > BST2 showed a higher sensitivity than $\mathrm{CD} 3^{+} \mathrm{tSV}$ to the inhibition provided by the MG132-induced depletion of free-ubiquitin $[5,20,21]$, suggesting that MVB-associated stores are unlikely to be affected by the acute 
pharmacological inhibition of the ESCRT machinery. The significant reduction of $\mathrm{CD}_{40 \mathrm{~L}}^{+}$ and $\mathrm{CD} 3^{+} \mathrm{tSV}$ transfer following N-SMases inhibition likely results from interference with broader membrane trafficking events involving both the TGN and endosomes where NSMases preferentially locate to regulate membrane curvature and budding [22, 23]. Finally, our data suggest that compared to other vesicle subpopulations, the production of $\mathrm{CD}_{40 \mathrm{~L}^{+}}$ tSV requires the stepwise transport of CD40L to the PM and budding SE (Fig. S3G,H,J). While BFA and manumycin inhibited the $\alpha$-CD3-induced upregulation of CD40L, CytD impaired CD40L ${ }^{+} \mathrm{tSV}$ release despite its effective cell surface upregulation (Fig. S3D and $\mathrm{S} 3 \mathrm{H})$.

\section{T cell subsets have distinct tSV cargo.}

Next, we sought to evaluate whether different human $\mathrm{T}$ cells, including $\mathrm{CD} 8^{+}$ cytotoxic $\mathrm{T}$ cells (CTL), $\mathrm{CD}^{+} \mathrm{T}$ helper cells $(\mathrm{TH})$ and cultures enriched in $\mathrm{CD} 127^{\text {low }} \mathrm{CD} 25^{\text {high }}$ FoxP $^{+}$cells (Treg), displayed distinctive TV transfer hallmarks. All cells were isolated from peripheral blood, activated, and further expanded ex vivo as detailed in Methods (see Fig. S4A-C). We evaluated the transfer of the TCR $\alpha \beta$ heterodimer, CD2, the coreceptors CD4 or CD8, CD28, CD45, the tSV proteins CD63, CD81 and BST2, and effectors including regulatory enzymes (CD38, CD39 and CD73), and helper (CD40L) and cytotoxic (Perforin) proteins. Analyses were focused on the geometric mean or median fluorescence intensities of the population of single $\mathrm{T}$ cells and single BSLB after gentle dissociation of conjugates (Fig. 2A). A normalised synaptic transfer metric was defined as the percent enrichment of $\mathrm{T}$ cell proteins on single BSLB from the total mean signal observed in single BSLB and single cells (Fig. 2A and ref.[5]).

Because we reconstituted our BSLBs in the absence of ligands for TCR coreceptors CD4/CD8 (i.e., pHLA) and CD2 (i.e., CD58), we therefore expected the vesicular shedding 
of TCR to include limited coreceptors and CD2 (Fig. 2B-D). Compared with TH, CTLs transferred significantly more TCR and coreceptor in the range of $\alpha$-CD3e-Fab densities tested and consistently showed smaller $\alpha$-CD3e-Fab $\mathrm{EC}_{50}$ values of 251.2 for $\mathrm{TCR}$ (compared with 1,251 in TH) and 50.87 for the coreceptor (compared with 56.44 in $\mathrm{TH}$ ) (Fig. 2B-D). The higher antigen sensitivity of CTL compared with TH and increased physical proximity between TCR and CD8 [24], might promote their sorting in tSV. Similarly, using chimeric antigen-receptor expressing T cells (CART) specific for HLA-A*02: NY-ESO- ${ }_{157-}$ 165 we tested whether the T1 CAR displays similar transfer dynamics than TCR. Interestingly, we observed a significantly higher transfer of the CAR than TCR at comparable densities of $\alpha-C D 3 \varepsilon-F a b$ and agonistic HLAp complexes (Fig. S4D). CART also transferred higher amounts of CD8, suggesting that CAR triggering by agonistic pHLA, even at low densities, promotes the efficient release of CAR and CD8 (Fig. S4E). Like CD2 and coreceptors, and despite their high level of cell surface expression, we observed a lack of CD28 and CD45 enrichment in the tSV released by different $\mathrm{T}$ cell subsets (Fig. 2C-F). While CD45 is normally excluded from the cSMAC and the synaptic cleft, the coreceptors, CD2 (at exceptionally low levels) and CD28 require binding to cognate ligands to be mobilised as microclusters to the synapse centre [25-27]. The latter suggests that tSV heterogeneity is partly defined by APC ligands dynamically feeding information to T cells, which we explored further in the next section. The limited enrichment of CD4, CD8, and CD45 in tSV makes them suitable secondary parameters for discriminating single BSLBs in FCM analyses and sorting (Figs. S1A, S4I, and S6A).

$\mathrm{TH}, \mathrm{CTL}$, and Treg transferred comparable levels of $\mathrm{CD}^{+} 3^{+}, \mathrm{CD} 1^{+}$, and $\mathrm{BST} 2^{+} \mathrm{tSV}$ to BSLB (Fig. 2G-I), indicating conserved mechanisms delivering these components to the synaptic cleft. Consistently, CART showed a comparable transfer of $\mathrm{CD}^{+} 3^{+} \mathrm{tSV}$ when stimulated through the CAR and the TCR (Fig. S4F). We next measured the vesicular 
transfer of effector ectoenzymes, namely the ADP-ribosyl cyclase CD38 and the AMP- and adenosine-producing ectonucleotidases CD39 and CD73 (Fig. 2J-L). While CD38 polarises to the IS[28] and participates in the generation of T-dependent humoral immunity[29], CD39 and CD73 are known tolerogenic mediators [30-32]. TH and CTL showed a limited, conserved, and comparable transfer of $\mathrm{CD}^{+} 8^{+} \mathrm{tSV}$ to BSLB (Fig. 2J). Treg, on the other hand, showed an increased transfer of CD39 $\left(\mathrm{T}_{\max }=17.83 \%\right.$ compared with $6.5 \%$ in $\mathrm{TH}$; Fig. $2 \mathrm{~K}$ ), and CD73 ( $\mathrm{T}_{\max }=5.73 \%$ compared with $1.9 \%$ in $\mathrm{TH}$; Fig. 2L), which associated with a higher central clustering of CD39 and CD73 in the cleft of Treg synapses (Fig. S4J-K). The inclusion of itinerant enzymes such as CD38, CD39, and CD73 in tSV, although limited, might exert a feed-forward regulatory effect as shown for tumor-derived sEV[33].

We also compared the transfer dynamics of CD40L and perforin, two major effectors mediating the activation and killing of APCs, respectively. Upon TCR triggering, we observed a conserved relative transfer of CD40L in T cells, with TH secreting the most (Fig. 2M, see also Fig. 3). Conversely, compared to TH, CTLs showed a reduced threshold for perforin release, which was transferred to BSLB at $\alpha$-CD3 $\varepsilon-F a b$ densities as low as 8 molec. $/ \mu \mathrm{m}^{2}$ for $\mathrm{CD}^{+} \mathrm{T}$ cells (Fig. $2 \mathrm{~N}$ ). In CART cells, perforin release followed a different behaviour than exocytic $\mathrm{CD}^{+} 3^{+} \mathrm{tSV}$. More specifically, TCR-elicited perforin deposition on BSLB was significantly higher than that resulting from CAR-triggering (Fig. S5D), suggesting differential mechanisms influencing the release of tSV and cytotoxic SMAPs at the IS. Altogether, our data indicate that 1) T cell subsets display differential dynamics of tSV transfer consistent with their effector functions and receptor-ligand interactions occurring at the IS and that 2) BSLB are excellent tools to study the biogenesis of both tSV and perforin-containing SMAPs.

\section{IS composition regulates SV cargo.}


In the dynamic exchange of information between $\mathrm{T}$ cells and APCs several factors might influence the effector cargo of tSV. Hence, we harnessed BSLB to study the dynamics influencing $\mathrm{CD}_{40 \mathrm{~L}^{+}} \mathrm{tSV}$ release under near-physiological conditions and using absolute quantifications (please refer to Methods). Absolute measurements offer a better comparison of varying CD40L expression levels among different T cell subsets (e.g., Fig. 2M). First, we measured $\mathrm{CD}_{40 \mathrm{~L}^{+}} \mathrm{tSV}$ release in response to $\mathrm{CD} 40$ densities of different primary human $\mathrm{B}$ cell subsets (minimum and maximum values of 16 and 646 molec./ $\mu \mathrm{m}^{2}$; Fig. 3A). BSLB covering a range of 0 to 540 molec./ $/ \mathrm{m}^{2}$ of $\mathrm{CD} 40$ were used to compare the $\mathrm{tSV}$ transfer dynamics to BSLB between previously activated (and expanded) $\mathrm{TH}$ blasts and freshly isolated (quiescent) TH. As shown in Fig. 3B, while quiescent TH transferred negligible amounts of CD40L, activated $\mathrm{TH}$ showed high sensitivity to CD40, transferring CD40L to CD40 densities as low as 2 molec./ $\mu \mathrm{m}^{2}$. However, this high efficiency reached a maximum transfer $\left(\mathrm{T}_{\max }\right)$ at around 20 molec./ $\mu \mathrm{m}^{2}$ of $\mathrm{CD} 40$, which at higher densities outcompeted the anti-CD40L detection antibody [5]. The latter is expected as CD40 displays a high affinity for its ligand (0.5-7.13 $\mathrm{nM}$ [34]). Therefore, we used the physiological minimum of CD40 (20 molec. $/ \mu \mathrm{m}^{2}$ ) to study the dynamics affecting CD40L sorting in $\mathrm{tSV}$. We used a vesicle stamp area of $0.84 \mu \mathrm{m}^{2}$ [5] to estimate and compare the effective densities of CD40L on the vesicular stamps of BSLB. Even after $4 \mathrm{~h}$, no significant transfer of vesicular CD40L was observed from quiescent cells (Fig. 3C). In contrast, activated TH blasts transferred significant amounts of CD40L and led to densities up to 1,000 times those found on the PM, suggesting a significant gain in CD40L binding valency resulting from its vesicular packing. Remarkably, higher $\mathrm{CD}_{40 \mathrm{~L}^{+}} \mathrm{tSV}$ transfer in activated cells related to their higher expression of TSG101 (Fig. 3D), indicating that T cell activation leads to the simultaneous up-regulation of effectors and ESCRT-I. Further comparison of TH, Treg, and CTL revealed phenotypespecific differences in the dynamics of CD40L transfer among activated $\mathrm{T}$ cells, with the 
amounts released in the order TH>Treg $>$ CTL (Fig. 3E). As in Fig. 2L, TH showed increased sensitivity for CD40L release compared with Treg and CTL (F-test, p<0.0001).

Next, we addressed whether TCR:pMHC binding potency influences the release of $\mathrm{CD}_{40 \mathrm{~L}^{+}} \mathrm{tSV}$. We used $3 \mathrm{TH}$ clones specific for the same influenza H3 hemagglutinin peptide HA $_{338-355}$ (NVPEKQTRGIFGAIAGFI) but displaying different TCR:pMHC potencies as determined by an IFN- $\gamma$ release assay (Table S2). T cell clones showed comparable levels of CD40L transfer at higher antigenic HLA densities and formed morphologically similar synapses (Fig. 3D-E). However, contrary to their IFN- $\gamma$ response, clones showed differences in the $\mathrm{EC}_{50}$ for $\mathrm{CD} 40 \mathrm{~L}$ transfer with clone 40 displaying the lowest $\left(P=0.0002, \mathrm{EC}_{50}=22.02\right.$, compared to 66.01 and 101.9 for clones 16 and 35, respectively; Table S2), suggesting that TCR: pMHC affinity has more substantial effects on the release of $\mathrm{CD}_{40 \mathrm{~L}^{+}} \mathrm{tSV}$ at lower antigen densities. The latter phenomenon might underpin the maintenance of an active CD40L-CD40 pathway in autoimmune diseases, where high densities of self-antigens might suffice to trigger the release of $\mathrm{CD} 4 \mathrm{~L}^{+} \mathrm{tSV}$ despite low affinities of TCR for self-peptides and the assembly of non-canonical immune synapses [35].

Beyond antigens (signal one), APCs also present membrane-bound accessory signals (signal two) to fine-tune the activation of engaged T cells. Because the influence of accessory molecules on the vesicular output of the synapse is not fully understood, we next sought to evaluate the effects of different known $\mathrm{T}$ cell costimulatory and repressing signals on the shedding of CD40L ${ }^{+}$and other $\mathrm{tSV}$. We incorporated costimulatory signals including CD80, CD86, ICOSL, and CD58, the latter of which forms a distal domain boosting TCR signalling known as CD2 corolla [27]. We also included the negative regulators PD-L1 and PD-L2 and other TNF ligand superfamily (TNFSF) members, including OX40L (TNFSF4) and 4-1BBL (TNFSF9), whose interplay with the CD40-CD40L dyad remains poorly characterised. To evaluate whether the synaptic output of $\mathrm{T}$ cells could also be affected by foreign proteins, 
such as those participating in virological synapses, we included the HIV-1 gp120 protein, which is a known high-affinity ligand for CD4 [26, 36-38]. We then compared the differences in NST\% between different BSLB compositions using those containing only ICAM1, CD40, and a titration of $\alpha-C D 3 \varepsilon-F a b$ as controls (Fig. 3H-P). While CD58 incorporation slightly promoted transfer of CD2 (Fig. 3I), incorporation of CD80, CD86 and ICOSL induced an increased synaptic transfer of CD28 (Fig. 3J), and in a lesser extent that of CD40L (Fig. 3M). Most remarkably, the inclusion of PD-L1 and PD-L2 on BSLB resulted in a significant reduction in the transfer of $\mathrm{CD} 40 \mathrm{~L}^{+} \mathrm{tSV}$ (Fig. 3M), and a less significant reduction of $\mathrm{TCR}^{+}, \mathrm{CD} 28^{+}, \mathrm{CD}^{+} 3^{+}, \mathrm{CD} 1^{+}$, and $\mathrm{BST}^{+} \mathrm{tSV}$. Similarly, BSLB loaded with a combination of 4-1BBL and OX40L impaired the transfer of vesicular TCR, CD81, CD40L, and BST2 from TH (Fig. 3H, L-N). Whether these differences relate with the competitive occupancy of intracellular signalling adaptors shared between CD40L, OX40 and 4-1BB are subject of further investigation. BSLB loaded with the HIV-1 gp120 instigated a reduced transfer of $\mathrm{TCR}^{+}, \mathrm{CD}^{+} 3^{+}$and $\mathrm{CD} 81^{+} \mathrm{tSV}$ at high $\alpha-\mathrm{CD} 3 \varepsilon$ Fab without significantly affecting the transfer of CD40L ${ }^{+}$tSV (Fig. 3H, K-M). Importantly, gp120 promoted CD4 transfer to BSLB (Fig. 3P) even without TCR triggering. As previously shown, CD4 transfer might be

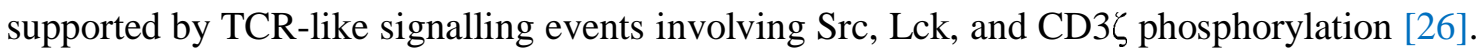
The gp120-induced transfer of CD4 and the CD80/86-instigated transfer of CD28 provide further evidence linking ligand binding with the sorting of proteins in tSV. Altogether these data provide proof of concept that as synthetic APCs, BSLBs facilitate the study of cognate and non-native molecular interactions occurring at the cell-bead interface and shaping the composition of $\mathrm{T}$ cell $\mathrm{tSV}$.

Next, we used CRISPR/Cas9 genome editing to test the functional relevance of endogenous elements participating in tSV biogenesis and the inclusion of CD40L. Because we used anti-CD3 Fab for the HLA-independent triggering of TCR, we expected negligible 
participation of CD4 in tSV biogenesis. Hence, we selected a guide RNA (gRNA) producing downregulation of the coreceptor $(67.83 \pm 10 \%$ of baseline $)$ as a control. We also selected gRNAs targeting membrane structural proteins $(B S T 2, C D 81)$ and the ESCRT-I component TSG101, which participates in the budding of SE [5]. We also targeted ADAM10, a disintegrin and metalloproteinase mediating the shedding of surface FasL and CD40L from activated T cells $[39,40]$, as it might mediate CD40-induced transfer of soluble CD40L trimers [41]. Knockout of the relevant targets was observed with some residual surface on cells edited for BST2 (45.08 $\pm 23.19 \%$ of control), CD81 (7.63 $\pm 4.8 \%$ of control), and ADAM10 (9.37 $\pm 2.83 \%$ of control). ADAM10 downregulation led to a significant increase of cell surface CD40L $(224.2 \pm 114.8 \%)$ (Fig. S5A-D). We compared tSV transfer in terms of the $\mathrm{T}_{\max } \%$ observed in controls (CD4-gRNA). While ADAM10 downregulation increased both the cell surface expression and the transfer of $\mathrm{CD}_{40 \mathrm{~L}^{+}} \mathrm{tSV}$ to BSLB, the downregulation of CD81, BST2 and TSG101 reduced the transfer of $\mathrm{CD}_{40 \mathrm{~L}^{+}} \mathrm{tSV}^{\mathrm{S}}$ without altering the baseline expression levels of CD40L at the PM (Fig. 4A and S5A-B). As expected, CD81 and BST2-edited cells showed significant reduction in the transfer of their respective $\mathrm{CD} 1^{+}$and $\mathrm{BST}_{2}{ }^{+} \mathrm{tSV}$ (Fig. 4D-E, respectively), and non-significant differences with regards to other markers (Fig. 4B-E). TSG101 editing led to a significant reduction of $\mathrm{CD} 4 \mathrm{~L}^{+}$and $\mathrm{TCR}^{+} \mathrm{tSV}$ transfer without affecting their baseline surface expression levels, indicating a bona-fide inhibition of their vesicular sorting dependent on ESCRT-I (Fig. 4AB). TSG101 downregulation partly phenocopied the effects of ubiquitin depletion by MG132, with inhibition of transfer affecting mostly CD40L $\mathrm{L}^{+} \mathrm{tSV}$, and to a lesser extent $\mathrm{TCR}^{+} \mathrm{tSV}$ (Fig. 2C-D). $\mathrm{CD}^{+} 3^{+} \mathrm{tSV}$ transfer was less affected by downregulation of CD81, ADAM10, BST2 and TSG101 (Fig. 4C), providing an additional piece of evidence for CD63 vesicular transfer relating mostly to the transport and exocytosis of preformed MVB, which is also congruent with our observations with the panel of inhibitors. We next sought to evaluate 
whether reduced tSV transfer to BSLB relates with impaired trafficking and central clustering of CD40L at the T cell-SLB interface. We compared CD40L total FI within synapses (i.e., as median IntDen per donor) as a function of the Tmax of controls. Imaging of ISs formed on planar SLB further revealed that reduced transfer to BSLB correlated with a significant reduced total clustering of CD40L within synapses of CD81- and TSG101-edited cells, with a less stronger effect observed in BST2-edited cells (Fig. 4F-G). When analysing the dynamics of $\mathrm{tSV}$ transfer to BSLB we observed a reduced $\mathrm{T}_{\max }$ of $\mathrm{CD} 40 \mathrm{~L}^{+} \mathrm{tSV}$ for CD81-, BST2-, and TSG101-edited cells, the latter of which also displayed a reduced $\mathrm{T}_{\max }$ for $\mathrm{TCR}^{+} \mathrm{tSV}$ (Fig. S5F-I). TSG101-edited cells also showed reduced upregulation of cell surface CD40L upon TCR-triggering (Fig. S5J), consistent with the reduced total clustering of CD40L at the IS interface. Expectedly, ADAM10-edited cells showed increased $\mathrm{T}_{\max }$ and reduced $\alpha$-CD3ع-Fab $\mathrm{EC}_{50}$ for the release of $\mathrm{CD} 40 \mathrm{~L}^{+} \mathrm{tSV}$, and a marked cell surface upregulation of CD40L following $\alpha-C D 3 \varepsilon$ stimulation (Fig. S5I and J, respectively). The partial reduction in CD40L transfer following downregulation of CD81 and BST2, suggests that rather than being essential, these integral membrane proteins work as aiding factors in the release of CD40L ${ }^{+}$, but not $\mathrm{TCR}^{+}$nor $\mathrm{CD}^{+} 3^{+} \mathrm{tSV}$. Also, the differential requirements for TSG101 among $\mathrm{TCR}^{+}$, $\mathrm{CD} 4 \mathrm{~L}^{+}$and $\mathrm{CD}^{+} 3^{+} \mathrm{tSV}$, which is phenocopied by MG132 treatment, demonstrates different ESCRT-I requirements among tSV. The increased transfer of $\mathrm{CD}_{40 \mathrm{~L}^{+}} \mathrm{tSV}$ from ADAM10deficient cells also suggests minimal inclusion of cleaved ectodomains in the transferred pool of CD40L, which is also supported by our immunoblot analyses (Fig. S1G-H).

\section{tSV carry distinct miRNAs from EVs.}

Within the framework of harnessing BSLB to study particulate effectors released at the IS, we then sought to identify miR species associated with $\mathrm{tSV}$. To minimize cell contamination, we isolated BSLB coated with $\mathrm{TCR}^{+} \mathrm{tSV}$ using fluorescence-activated cell 
sorting of single beads after 90 min of culture with T cells (Fig. S6A), followed by small RNA purification. Small RNAs were also purified from EVs from the same donors as controls. Prior to comparing the RNA species identified in cells, EVs and tSV, we used the less abundant RNA species found in null BSLBs (Fig. S6A, grey overlaid histogram) as background for the identification of miR enriched in tSV. Analyses of tSV and EVs libraries' raw reads revealed a similar composition of small RNA species, of which miRs were overall more enriched in tSV than EVs and the parental cells (Fig. 5A). After identifying small RNA species conserved across samples, donors, and sequencing runs, GSEA further revealed public and tSV- or EVs-specific miRs in both TH and CTL. Of $200 \mathrm{miR}$ species enriched in $\mathrm{TH}, 11$ were uniquely enriched in tSV and $23 \mathrm{in} \mathrm{EVs,} \mathrm{whereas} \mathrm{of} 163 \mathrm{miR}$ species enriched in CTL, 17 were unique to tSV and 19 to EVs (Fig. 5B and Table S3). Consistent with the wider variety of enriched miR in sEV, we observed a higher variance compared to tSV (Fig. 5C). Analyses of enriched motifs with MEME [42] identified motifs to unique and shared miR species in TH (Fig. 5D) and CTL (Fig. 5E), which together redundantly associated with GO pathways related to immune cell signalling and anti-tumor immunity (Fig. 5F and heatmaps in Fig. S6B). Congruently, miR target enrichment analyses of enriched miR further revealed a similar degree of redundancy in the identified targets (see Table S4 for the full list of targets and Fig. S6C-H for GO terms), which included RNA-binding proteins, such as AGO1/2, DICER, GRB2, GRAP2, PLAGL2, RRM2, TNRC6A/B, and YBX1; immune receptor adaptors and signalling proteins such as CD2AP, IFNARs, SMADs; and synaptic ligands and effectors including ICAM1 and thrombospondin-1 (THBS1), among others.

Although EVs showed slightly more miR species, the overall percent enrichment of these small RNA species was higher in tSV. We next sought to evaluate whether the increased percent enrichment of miR in $\mathrm{tSV}$ relates to a higher RNA binding protein (RBP) content in these vesicles. Following protein capture, digestion and elution using S-Trap, we 
performed label-free quantifications with liquid chromatography-tandem mass spectrometry (LC-MS/MS) on tSV and EVs isolated by differential centrifugation of BSLB eluates and cell-cleared culture supernatants, respectively. Since contaminants derived from the elution reagents and procedure might impact the quality of our comparisons, we first identified proteins enriched in eluates derived from activating $\left(\alpha-\mathrm{CD} 3 \mathrm{Fab}^{+}\right)$BSLB comparing to null BSLB (coated only with ICAM1 and CD40)(Fig. S7A). With the aid of Pegasus we generated a list of differentially expressed proteins to compare with the EVs from the same TH and CTL cells, respectively (Fig. S7B, C). PANTHER based GSEA revealed vesiclespecific differences between eluates (tSV) and EVs, including the reduced detection of ADAM10 and contaminants Histone 3, LGALS3BP [43], and RNAse 4 in tSV (Fig. S7D), and the high enrichment of targets predicted to interact with tSV miRs, such as SMAD4, DNMT1 and GRB2 in tSV (Fig. S7E and Table S4). Proteins related to global nucleic acidbinding, including RNA-binding and RNA-interference, stood out in tSV compared with EVs (Fig. S7F, G), with an overall difference in the enriched proteins found in $\mathrm{tSV}$ and EVs. Among them, YBX1, a known protein packing small noncoding RNAs (sncRNA) in EVs $[44,45]$ was identified as a common RNA-binding protein enriched in tSVs (TH>CTL; Fig. S7F), as well as with > 3-fold enrichment in SE tethered to sorted BSLBs (see ref. [5]). Other RNA-binding proteins were also commonly identified in independent LC-MS/MS runs of eluted tSV and subpopulations of SE anchored to sorted BSLB (Fig. S7H). To validate these findings, we then performed TIRFM imaging of TH cell synapses to localise the distribution of some of these RBPs. The localization of in YBX1 and, in a lesser extent, the SE-enriched SF3B3[5] in both the synaptic cleft and the proximal membrane of T cells interacting with $\alpha$ CD3 $\varepsilon$ further validated our LC-MS/MS findings (Fig. S7I and J, respectively, compare with isotype control in $\mathrm{K})$. YBX1 localised as discrete puncta both in the synaptic cleft and in vesicular tracks left behind by cells spontaneously breaking synapse symmetry and resuming 
migration (compare with 7I, white arrows). Supporting the active release of vesicular RNA, we further found the polarisation of ER-like structures and release of ceramide ${ }^{+}$and $\mathrm{RNA}^{+}$ puncta in both the synaptic cleft and in vesicular tracks of live, non-permeabilised TH IS (Fig. S7L,M, yellow arrows). As evidenced by the super-resolution imaging with eTIRFSIM, most RNA puncta lined the periphery of the synaptic cleft as early as 5 minutes during synapse assembly, indicating the rapid mobilisation of $\mathrm{RNA}^{+}$compartments to the contacting membrane of stimulated $\mathrm{T}$ cells (Fig. S7M, right panel). The second layer of information provided by transferred miR and their protein partners might be pivotal for the functional modulation of APCs and the fine tuning of T cell activation and effector function by serving as a mechanism for suppressor miR elimination. Altogether, the polarisation and central clustering of immune receptors, ER-like structures, RNA and YBX1 to the synaptic cleft enriched in trans-synaptic effectors supports the superior role of the IS in the transfer of a broad range of intercellular messengers.

\section{DISCUSSION}

The first observation of $\mathrm{T}$ cells being successfully activated by bead-supported bilayers presenting antigenic MHC-II dates back to 1986 [46]. Others have demonstrated the versatility of BSLB as APCs in several experimental contexts, including measuring the dimensions of interfacial receptor-ligand interactions and the localisation of lipid species and CD3 $\varepsilon$ in the interacting pole of T cells $[47,48]$. Here we leveraged the APC capabilities of BSLB to isolate and study in more detail the composition and biogenesis of T-cell released EVs. Most importantly, BSLB facilitated novel comparisons of tSV and EVs, providing evidence for highly heterogeneous yet unique populations of vesicles being released in the limited dwell-time of the IS. We corroborated quantitative differences in size, protein loads and association to miR and RBPs between tSV and EVs. Also, the rate of secretion is higher 
for tSV compared with EVs. We have previously shown that TH IS shed an approximate of 25-30 TCR $\alpha \beta^{+}$SE after 20 min of IS formation on SLB $[4,5]$. In contrast, the quantification of TCR $\alpha \beta^{+} \mathrm{EV}$ s by NanoFCM evidenced their rather low constitutive secretion ranging from a minimum of 0.54 to a maximum of $8.96 \mathrm{EVs} /$ cell over a period of $48 \mathrm{~h}$ of culture.

The acute pharmacological inhibition and CRISPR/Cas9-editing of TH strongly indicated that mechanistically $\mathrm{tSV}$ heterogeneity is partly explained by contributions of several cellular machineries recruited at the interacting pole of activated $\mathrm{T}$ cells. We provide evidence for an overarching hierarchy in the mechanisms participating in the vesicular transfer of CD40L, with dominant participation of ER/TGN transport and ESCRT machinery, and secondary involvement of MVB and lysosomes. The selective increase in $\mathrm{TCR}^{+} \mathrm{tSV}$ transfer resulting from dynamin inhibition and the reduced effect of ESCRTinhibition/downregulation on $\mathrm{CD}^{+} 3^{+} \mathrm{tSV}$ transfer provide additional evidence for different sorting mechanisms participating in the biogenesis of tSV (Figs. S3C-F and 5). We provide evidence for a critical role of ADAM10, a protease known to be active at the PM, in regulating the pool of surface CD40L and the resulting levels of CD40L ${ }^{+}$tSV shedding dependent on CD40. Interestingly, our LC-MS/MS analyses indicate that compared to tSV, EVs incorporate a higher amount of ADAM10 (Fig. S7D), explaining their reduced vesicular CD40L (Fig. 1H).

Microvilli lead the formation of effector membranous particles referred to as synaptosomes enriched in LFA-1, CD2 and cytokines [49]. Microvilli tips accumulate proteins including TCR, CD2 and CD4 $[49,50]$. Here we show that T cells transfer little vesicular CD2 and CD4 (Fig.1B and 2C,D), which increased at limited levels only upon ligation by CD58 and HIV-1 gp120, respectively (Fig. 3I and 3P). Also, TCR signalling, which is required for tSV biogenesis, is known to produce microvilli disassembly [50], suggesting that synaptosomes differ in origin and structure from ectosomes and contribute 
minimally to the vesicular output of the IS. Rather, synaptosome-like structures might mediate the negligible release of EV-like material to BSLB sustaining cell adhesion but devoid of antigen/ $\alpha-C D 3$ Fab (Fig. 1H).

TSV biogenesis is a highly dynamic process connecting the integration of signals presented by APCs with the particulate output of activated $\mathrm{T}$ cells. Earlier electron microscopy studies demonstrated budding of $\mathrm{TCR}^{+} \mathrm{SE}$ with partial polarisation of MVB and TGN in stimulated cells (figure $1 \mathrm{C}$ in $\operatorname{ref}[4]$ ). In these experimental settings, SLB lacked CD40, which as we later demonstrated, promotes quick mobilisation of CD40L to the PM and into budding SE as microclusters [5]. Here we provide evidence that shedding of $\mathrm{CD}_{40 \mathrm{~L}^{+}} \mathrm{tSV}$ requires $\mathrm{CD} 40$ on presenting BSLBs and its quick mobilisation from the ERGolgi. We provide evidence of other signals finely tuning tSV heterogeneity, including B7 receptors (CD80/86), PD-1 ligands, other TNFSF members, and non-native ligands. For instance, HIV-1 gp120 promoted the vesicular transfer of CD4 and CD40L at low physiological densities of $\alpha$-CD3 $\varepsilon$ Fab. Similarly, the non-native interaction between CARs and antigenic HLA complexes led to a remarkably efficient transfer of CAR as part of tSV and at the expense of its cell surface expression levels (Fig. S4D, red arrow). The superior vesicular shedding of CAR containing $\mathrm{CD} 3 \zeta$ signalling tails might result from rewired TCR signalling networks and the formation of multiple short-lived synapses [51]. Since reduced CAR expression might desensitise CART to new encounters with target cells, more research is needed to understand the impact of CAR release on the therapeutic effectiveness of adoptive $\mathrm{T}$ cell therapies.

The small RNA sequencing of BSLBs made possible the first side-by-side comparison of the miR cargo associated with tSV and EVs. We found that both miRs and RBPs were significantly more enriched in tSV than EVs (Fig. 5A and S7F,G). The high level of target redundancy found in the enriched miRs of both $\mathrm{tSV}$ and EVs provides an alternative 
mechanism supporting the effector role of miRs, especially when few copies of single species are transferred associated with EVs [52]. In other words, unlike immune receptors, which require gains in avidity to feed-forward activation signals to APCs, miRs might rely on redundancy to modulate the function of APCs. We found RBPs enriched in LC-MS/MS of tSV and SE, namely YBX1 and SF3B3, in the synaptic cleft of TH, implying the transfer of miR ribonucleoprotein complexes across these short-lived cellular contacts. Since miR targets participate in cell cycle regulation, senescence, and immune receptor signalling, the release of $\mathrm{tSV}$ might also promote the clearance of miR species controlling various aspects of the $\mathrm{T}$ cell effector response. Interestingly, telomeric binding proteins were also found among the enriched GO terms of tSV (rank 27 in Fig. S7G) and the differential expression analyses of SE proteins [5]. Whether the transfer of miRs, and to a lesser extent telomeres, in tSV contributes to the paracrine regulation of senescence, as preliminarily shown for APCs transferring telomeres [53], remains to be elucidated.

How sncRNAs associate with tSV is not fully understood. The recent identification of $\mathrm{N}$-glycosylated sncRNA species on the cell surface [54] suggests that if such modified species do exist, they might be transferred associated with the glycocalyx of synaptic ectosomes. Since we have observed the shedding of glycan-rich particles within the synaptic cleft $([5,6]$ and WGA puncta in Figs. S2B-I, 3G, S4K), we are studying whether glycoRNAs are loaded on the surface of tSV.

The intrinsic feed-forward function of tSV-enriched immune receptors and miRs, specifically and timely released in response to antigen is different in kind and structure to other signals integrated during early $\mathrm{T}$ cell activation. Therefore, we consider that the heterogeneity and effector diversity of the particulate output of T-cell synapses, including tSV and SMAPs, deserves its functional classification as signal four. We expect BSLB will help others in the high throughput study of other key factors driving signal four-dependent 
communications. Undoubtedly, the comparison of EVs and tSVs in other immune cells known to secrete highly heterogeneous EVs, such as DCs [55], or in cells using similar interfacial communications (e.g., $\mathrm{CD} 4 \mathrm{~L}^{+}$platelets and stromal cells secreting ADAM10 inhibitors) is needed to elucidate whether tSV are pivotal information units of cellular networks. Pathogen evolution has taken advantage of the cellular machineries mediating tSV budding. For instance, the HIV-1 Gag protein facilitates the transfer of viral information among interacting cells by hijacking components of the ESCRT system, mimicking the budding of SE $[4,56,57]$. We expect BSLBs will also help unravelling new pathogenic determinants hijacking vesicular messages across infected cell interfaces, broadening our understanding of 'trojan horses' driving infectious diseases [56].

\section{FIGURE LEGENDS}

Figure 1. As synthetic APC, BSLBs trigger synapse formation and the release of tSV by stimulated $\mathbf{T}$ cells. (A) Representative time-lapse confocal microscopy showing the interaction between $\mathrm{TH}$ and $\mathrm{BSLB}$ and the active transfer of $\mathrm{CD}_{40 \mathrm{~L}^{+}}$synaptic stamps to BSLB (white arrowheads), which are left behind after the interaction resolves. CD40L was tracked using $1 \mu \mathrm{g} / \mathrm{mL}$ of anti-CD40L clone 24-31 AF647. (B) FCM analyses of vesicular transfer to BSLB (teal histograms) from TH (grey histograms). BSLB were reconstituted with increasing densities of $\alpha-\mathrm{CD} 3 \varepsilon$ Fab and 200 molec./ $\mu \mathrm{m}^{2}$ of ICAM1 and 20 molec./ $\mu \mathrm{m}^{2}$ of CD40. Vertical lines indicates the mode of cells and BSLB in the condition with no $\alpha-C D 3$ Fab (null). (C) After conjugate separation by cold, tSV are released from BSLBs with the use of $50 \mathrm{mM}$ EDTA. (D) Elution results in the release of $\mathrm{TCR}^{+}, \mathrm{CD} 40 \mathrm{~L}^{+}$and $\mathrm{CD} 81^{+}$, and to a lesser extent $\mathrm{CD}^{+} 3^{+}$, from the surface of BSLB as measured by FCM staining comparing null 
BSLB (black histograms), with BSLBs prior to (green), and after elution (violet). (E) Transmitted electron microscopy of eluates reveals the presence of tSV (white arrows) and small soluble proteins. Scale bar= $200 \mathrm{~nm}$. (F) Representative NanoFCM analyses showing the detection of $\mathrm{TCR} \alpha \beta^{+}$and $\mathrm{CD} 81^{+}$events in isolated $\mathrm{EVs}$ (left panel), and the eluted material from Null BSLB (middle panel) and $\alpha-\mathrm{CD}^{+}$BSLB (tSV, right panel). (G) overlaid histograms showing TCR $\alpha \beta$ expression on $\mathrm{CD} 1^{+}$vesicles from EVs (yellow) and tSV (light blue) compared to double negatives (grey histogram). (H) Left: Immunoblot for comparison of CD40L, CD81, Alix and TSG101 in the EVs or eluted tSV deriving from $5 \times 10^{6}$ of either cells or eluted BSLB, respectively. BSLB were previously cocultured with TH cells at 1:1 ratio. Whole cell lysate represents a total of $2.25 \times 10^{5}$ cells. Right: CD40L levels in eluted fractions (tSV) and steadily released EVs as measured by immunoblot. Shown is CD40L corrected MFI (cMFI) normalised to TSG101 cMFI. Normality was determined using Shapiro-Wilk test and statistical significance was determined by two-tailed unpaired t-test (D) and by unpaired, two-tailed Mann-Whitney test (H). ${ }^{*} \mathrm{p} \leq 0.05,{ }^{*} * \mathrm{p} \leq 0.005, * * * \mathrm{p} \leq 0.0005$, $* * * * \mathrm{p} \leq 0.0001$ and $\mathrm{ns}=$ not significant. Data represents mean $\pm \mathrm{SEM}$ of $\mathrm{n}=2(\mathrm{~A}, \mathrm{~F}, \mathrm{G})$ and $\mathrm{n}=4$ independent experiments (B-E, H).

Figure 2. The synaptic transfer of vesicular effectors relates to the functional properties of different $\mathbf{T}$ cell subsets. (A) Gating strategy for selecting single BSLB and T cells to measure the transfer of vesicular effector at the IS. BSLBs presenting $\alpha$-CD3e Fab (0 to 1,000 molec. $\left./ \mu \mathrm{m}^{2}\right), 200$ molec. $/ \mu \mathrm{m}^{2}$ of ICAM1 and $20 \mathrm{molec} . / \mu \mathrm{m}^{2}$ of CD40 were used in all experiments. (B-L) Percent Normalized Synaptic Transfer $\left(\mathrm{NST} \%=\left[\mathrm{cGMFI}_{\mathrm{BSLB}} /(\mathrm{cGMFI}\right.\right.$ BSLB $\left.\left.+\mathrm{cGMFI}_{\text {Cells }}\right)\right]^{* 100)}$ to BSLB from TH (green), Treg (blue) and CTL (violet). Ligands included (B) the antigen receptor heterodimer TCR $\alpha \beta$; (C) CD2; (D) TCR co-receptors (CD4 or CD8); (E) CD28; (F) CD45; (G) CD63; (H) CD81; (I) BST2; (J) CD38, (K) CD39; (L) 
CD73, and (M) CD40L. (N) Perforin (PRF1) transfer as background corrected GMFI is shown (null BSLB-subtracted). Representative histograms for most makers are shown on the right for TH (green) and CTL (violet). Statistical significance was determined by multiple ttest comparing tSV transfer across different $\alpha-\mathrm{CD} 3 \varepsilon$ Fab densities and among different $\mathrm{T}$ cell populations. $\alpha$-CD3-Fab $\mathrm{EC}_{50}$ and the marker transfer maximum $\left(\mathrm{T}_{\max }\right)$ were determined using F-test and three to four parameters fitting (H-J). ${ }^{*} \mathrm{p} \leq 0.05, * * \mathrm{p} \leq 0.005, * * * \mathrm{p} \leq 0.0005$, $* * * * \mathrm{p} \leq 0.0001$ and $\mathrm{ns}=$ not significant. Data represent means \pm SEM of $\mathrm{n}=4-10$ independent experiments.

Figure 3. BSLBs reveal the dynamics influencing the biogenesis of $\mathrm{CD}^{4} \mathrm{~L}^{+} \mathrm{tSV}$. (A) CD40 densities on the surface of human B cells isolated from either peripheral blood (CD19 ${ }^{\text {hi }}$ $\mathrm{HLA}_{-} \mathrm{DR}^{+}$and $\mathrm{CD} 40^{+}$) or palatine tonsils (also CXCR5 ${ }^{\mathrm{hi}}$ ). (B) Based on data in A, BSLBs were reconstituted with increasing densities of human CD40 and analysed for their capacity to instigate the release of $\mathrm{CD} 40 \mathrm{~L}^{+} \mathrm{tSV}$. Quiescent and $\alpha-\mathrm{CD} 3 / \mathrm{CD} 28$-activated $\mathrm{TH}$ are compared. BSLBs presenting either no $\alpha-\mathrm{CD} 3 \varepsilon$ or 1,000 molec./ $\mu \mathrm{m}^{2}$ were used in these experiments. (C) As in B, TH cells were incubated for $4 \mathrm{~h}$ with BSLB and the density of CD40L compared to $1 \mathrm{~h}$ activated TH controls. (D) TSG101 expression by flow cytometry (left) and immunoblotting in quiescent and activated TH. (E and F) Comparison of CD40L ${ }^{+}$ tSV transfer among different T cell types (green: TH; blue: Treg-enriched; violet: CTL), and among $\mathrm{T}$ cell clones (E) responding to the influenza $\mathrm{H} 3 \mathrm{HA}_{338-355}$ peptide presented in HLADRB1*09:01. (G) TIRFM images showing CD40L clustering within the synapses of $\mathrm{T}$ cell clones stimulated on SLB containing $30 \mathrm{molec} . / \mu \mathrm{m}^{2}$ of antigen, $200 \mathrm{molec} . / \mu \mathrm{m}^{2}$ of ICAM1 and 20 molec. $/ \mu \mathrm{m}^{2}$ of CD40. Scale bar $=5 \mu \mathrm{m}(\mathrm{A}-\mathrm{H})$ After cell: BSLB conjugate dissociation FCM measurement was performed on BSLB to calculate the NST\% of tSV positive for (H) TCR, (I) CD2, (J) CD28, (K) CD63, (L) CD81, (M) CD40L and (N) BST2 
was measured on control (green) or BSLB presenting CD80, CD86, ICOSL (blue), CD58 (red), PD-L1 and PD-L2 (magenta), HIV-1 gp120 (grey), and OX40L and 4-1BBL (black) (all at 100 molec./ $/ \mathrm{m}^{2}$ ). (O) Representative half-overlaid histograms showing $\mathrm{CD} 40 \mathrm{~L}^{+} \mathrm{tSV}$ transfer to ligand- and $\alpha-C D 3 \varepsilon-F a b-c o a t e d$ BSLB. Control BSLB were tailored to present

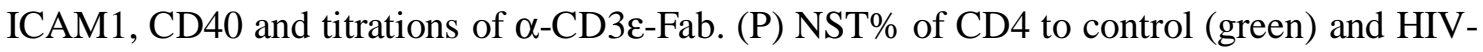
1 gp120-presenting BSLBs (grey; red arrows indicate the loss of CD4 on TH). Normality was determined using Shapiro-Wilk test, and statistical significance was determined by multiple ttest to compare levels of $\mathrm{CD} 40 \mathrm{~L}^{+} \mathrm{tSV}$ transferred to BSLB across different CD40 densities, times, $T$ cell populations (B-C, E-F), and increasing antigen densities (either $\alpha-C D 3 \varepsilon$ Fab or HLA-DRB $1 * 09: 01:$ HA complexes). $\alpha$-CD3e-Fab $\mathrm{EC}_{50}$ and marker maximum transfer $\left(\mathrm{T}_{\max }\right)$ were calculated using three to four parameters F-test (E, F). In D, Wilcoxon matched pairs signed rank test. $P=0.0312$ was used to compare TSG101 expression between quiescent and activated cells. In H-P, multiple t-test comparing control BSLB presenting 200 molec./ $\mu \mathrm{m}^{2}$ of ICAM1, 20 molec. $/ \mu \mathrm{m}^{2}$ of CD40 and $\alpha-C D 3 \varepsilon$ Fab (0 to 2,000 molec./ $\mu \mathrm{m}^{2}$ ) with BSLBs also presenting the selected group of proteins each at 100 molec./ $\mu \mathrm{m}^{2} .{ }^{*} \mathrm{p} \leq 0.05,{ }^{*} \mathrm{p} \leq 0.005$, $* * * \mathrm{p} \leq 0.0005, * * * * \mathrm{p} \leq 0.0001$ and $\mathrm{ns}=$ not significant. Data represents means \pm SEM of $\mathrm{n}=4$ (A-E and $\mathrm{H}-\mathrm{P})$ and $\mathrm{n}=3(\mathrm{~F}, \mathrm{G})$ independent experiments.

Figure 4. Harnessing BSLBs to identify endogenous proteins participating in the biogenesis of $\mathrm{CD40L}^{+} \mathrm{tSV}$. (A-E) Data showing the transfer of tSV as measured by flow cytometry of BSLB after the dissociation of cell:BSLB conjugates and expressed as percent of the maximum transfer $\left(\mathrm{T}_{\max } \%\right)$ observed in $C D 4$-edited controls. Heatmaps show $\mathrm{T}_{\max } \%$ for different tSV populations, including those positive for (A) CD40L, (B) TCR $\alpha \beta$, (C) CD63, (D) CD81, and (E) BST2 on BSLB incubated with different CRISPR/Cas9-edited cells (targets shown in italic). BSLB contained $200 \mathrm{molec} . / \mu \mathrm{m}^{2}$ of ICAM-1, $20 \mathrm{molec} . / \mu \mathrm{m}^{2}$ 


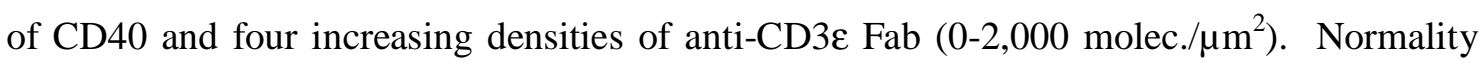
was determined using Shapiro-Wilk test and statistical significance was determined by multiple t-test corrected for multiple comparisons (Holm-Sidak) and comparing tSV transfer to BSLB between CRISPR/Cas9 edited cells and CD4-edited controls (depicted as \% of CD4-edited $\left.\mathrm{T}_{\max }\right) . \quad * \mathrm{p} \leq 0.05, * * \mathrm{p} \leq 0.005, * * * \mathrm{p} \leq 0.0005, * * * * \mathrm{p} \leq 0.0001$ and $\mathrm{ns}=$ not significant. (F-G) TIRFM imaging and quantification of total CD40L FI in the synapses formed by CRISPR/Cas9 genome-edited cells. (F) Median CD40L integrated density (i.e. total FI) in immunological synapses of CRISPR/Cas9-edited cells as \% of the maximum observed. Each circle represents a donor. Means of medians were compared using Mixedeffect analysis with geisser-greenhouse correction and Fisher's LSD test. (G) Top: Representative TIRFM images showing mature immunological synapses and the levels of centrally clustered CD40L. Bottom: diagram depicting the imaging of CD40L and other interfacial signals in IS formed between T cells and stimulating SLB. Data representative of $\mathrm{n}=6(\mathrm{~A}-\mathrm{E})$ and $5(\mathrm{~F}-\mathrm{G})$ independent experiments.

Figure 5: tSV are enriched in miRs, including both private and public species with considerable functional redundancy to EVs. (A) Abundance of different genome-mapped RNA species found associated with tSV, syngeneic steadily released EVs, and parental cells. (B) Venn diagram showing the overlap of enriched miR species associated with tSV, EVs and parental cells (see also Table S3). (C) miR heterogeneity is further reflected by PCA of the analysed tSV and EVs. (D-E) Motif analyses using MEME identified consensus sequences (central inserts) mapped across different miRs in TH tSV (D, top), TH EVs (D, bottom), CTL tSV (E, top) and CTL EVs (E, bottom). (F) Bar graph displaying the number of miR species enriched uniquely in tSV (violet), uniquely in EVs (green), or shared (grey). TH miR targets are shown in the top panel, and CTL miR targets are shown in the bottom panel. 
Data representative of at least six independent experiments, samples with low RNA yields were pooled for sequencing ( $\mathrm{n}=8$ donors).

\section{Supplementary Figure 1; related to Fig. 1. Multiparametric analyses of released tSV} show a highly heterogeneous population of vesicles being transferred at the IS. (A) T cell: BSLB synapses and the release of synaptic ectosomes containing engaged $\mathrm{T}$ cell molecules can be studied by conventional multicolour flow cytometry. Shown is the gating strategy to discriminate single BSLB and T cells following conjugate dissociation, and the biparametric overlays of single BSLBs either null (orange), agonistic (brown) and nonstimulated (light green) and TCR-stimulated cells (dark green) for BST2, CD63, TCR $\alpha \beta$ and CD38. (B) conventional FCM analyses of T cells after conjugate dissociation shows neligible uptake of fluorescent lipids by the stimulated T cells, which is observed across a broad range of $\alpha-\mathrm{CD} 3 \varepsilon$ Fab densities. Data shown represent the $\%$ of the total Atto 565 signal observed in null BSLB. (C) Nanoparticle Tracking analysis size distribution of tSV and EVs isolated by differential centrifugation. (D) using the size calibration beads (top left panel), EVs (green and top middle panel) tSV (violet and top right panel) were compared across different bin sizes (SS-A range) as defined by the CV of standard bead population. (E) Size distribution measured by NanoFCM of TCR, CD63, CD40L, BST2 and CD81 positive EVs (green) and tSV (violet). (F) Example overlaid histograms showing the comparison of TCR $\alpha \beta^{+}$vesicle size using benchmark calibration silica beads (grey). Compared TCR $\alpha \beta^{+}$EVs (yellow) and tSV (blue) were isolated by differential and ultracentrifugation as described in Methods. (G) NanoFCM for the comparison of TCR $\alpha \beta$ expression in EVs and eluted tSV. Expression was normalised to the signal measured in isotype-labelled controls (TCR $\alpha \beta$ MFI / Isotype MFI).

(H) Immunoblot showing the size comparison between tSV-associated CD40L and a recombinant human CD40L ectodomain. Null BSLB were loaded only with ICAM1 and 
CD40. Normality was determined using Shapiro-Wilk test and statistical significance was determined by Multiple paired t-test corrected by two-stage step-up method of Benjamini, Krieger and Yekutieli (FDR Q= 5\%)(D), by unpaired double tailed t-test (E) and by unpaired, two-tailed t-test $(\mathrm{G}) . P$ values are indicated above bars for the relevant comparisons. Data represent means \pm SEM from $n=4$ independent experiments.

\section{Supplementary Figure 2; related to Fig. 1. TIRFM and Airyscan confocal imaging of T} cell synapses indicate a high spatial segregation of T cell tSV. (A) Pearson's correlation coefficient (PCC) for the spatial colocalization between CD40L and other vesicle markers released within the cleft of TH synapses. Each dot represents the average PCC per synapse with representative TIRFM images shown in B-E. Glycans in the PM and tSV are labelled with Wheat Germ Agglutinin (WGA). SIRC: surface interference reflection contrast microscopy. Scale bar $=5 \mu \mathrm{m}$. (B-E) Representative TIRFM microphotographs showing the discrete spatial distribution of CD40L and other extracellular vesicle markers in nonpermeabilised, surface-stained TH synapses stained with monoclonal antibodies specific to CD40L and CD63 (B); CD81 (C); BST2 (D); and ADAM10 (E, as control). F-I) Airyscan confocal microphotographs showing the 3D spatial distribution of CD40L and other tSV markers, namely (F) CD63, (G) BST2, (H) TCR $\alpha \beta$, and (I) CD81 on synapses formed between TH and glass-SLB after 15 min of interaction. As a counterstaining we used WGA CF594, which labels cell surface glycans. Right panels: orthogonal views of TH synapses with the Z-plane spanning the synaptic cleft. Scale bar $=5 \mu \mathrm{m}$. In A-I, TH were stimulated on glass-supported lipid bilayers containing 200 molec./ $\mu \mathrm{m}^{2}$ of ICAM1, 20 molec./ $/ \mu \mathrm{m}^{2}$ of CD40 and 30 molec. $/ \mu \mathrm{m}^{2}$ of $\alpha$-CD $3 \varepsilon$ Fab. In (A) Normality was determined using Shapiro-Wilk test and statistical significance was determined by Kruskal-Wallis test corrected for multiple 
comparisons (Dunn's). Data representative of $\mathrm{n}=2$ independent experiments. $* \mathrm{p} \leq 0.05$, $* * \mathrm{p} \leq 0.005, * * * \mathrm{p} \leq 0.0005, * * * * \mathrm{p} \leq 0.0001$ and $\mathrm{ns}=$ not significant.

\section{Supplementary Figure 3; related to Fig. 1. The output from different subcellular} compartments underpins tSV heterogeneity. TH were preincubated for $30 \mathrm{~min}$ with a panel of 12 inhibitors and then co-cultured with control and agonist BSLB at a 1:1 ratio while keeping inhibitor concentrations. When possible, we used compounds redundantly targeting subcellular processes to elucidate effects related with the shared targets rather than to offtargets and used concentrations validated elsewhere [20, 21, 58-78] (Table S1). (A) After 90 min of culture, we measured the cell surface expression of key synaptic and tSV markers, and (B) the stability of cellular contacts by tracking the percent of remaining cell: BSLB conjugates following cold incubation. BSLB presenting 200 molec. $/ \mu \mathrm{m}^{2}$ ICAM1, 20 molec. $/ \mu \mathrm{m}^{2}$ of CD40 and increasing densities of anti-CD3 $\varepsilon$ Fab were used in all experiments. (C-F) Transfer of $\mathrm{TCR} \beta^{+}(\mathrm{C}), \mathrm{CD}^{+} \mathrm{L}^{+}(\mathrm{D}), \mathrm{BST}^{+}(\mathrm{E})$, and $\mathrm{CD}^{+} 3^{+}$(F) tSV to BSLB compared and normalised to the maximum transfer observed in control untreated cells (i.e., maximum measured transfer $\left(\mathrm{T}_{\max }\right)$ per donor and at 2,000 molec. $/ \mu \mathrm{m}^{2}$ ). Right heatmaps display $P$-values for the comparison of tSV transfer between controls and inhibitors, and across different $\alpha$-CD3e-Fab densities. (G-J) Dynamics of TCR $\alpha \beta(\mathrm{G}), \mathrm{CD} 40 \mathrm{~L}(\mathrm{H})$ BST2 (I) and CD63 (J) cell surface expression normalised to the baseline of untreated controls. In (J) out of range values (>200\% are represented in orange). Statistical significance and $P$-values were determined using mixed-effects model ANOVA with Geisser-Greeenhouse correction for the determination of normalised protein expression across different donors (A), and multiple t-test to compare frequency of conjugates (B), levels of $\mathrm{T}_{\max } \% \mathrm{tSV}$ transfer (C-F), and levels of normalised cell surface expression of relevant markers as \% of control baseline 
$(100 \%)(\mathrm{G}-\mathrm{J})$ across different $\alpha$-CD3 $\varepsilon$ Fab densities. ${ }^{*} \mathrm{p} \leq 0.05, * * \mathrm{p} \leq 0.005, * * * \mathrm{p} \leq 0.0005$, $* * * * \mathrm{p} \leq 0.0001$ and $\mathrm{ns}=$ not significant. $\mathrm{N}=6$ different donors.

Supplementary Figure 4; related to Figure 2. (A-C) Isolation and expansion of FoxP $3^{+}-$ enriched $\mathrm{cCD}^{+} \mathrm{T}$ cell cultures (termed herein as Treg). (A) sorting of cells on day 0 based on the high expression of CD4, negative expression of CD127 and further separation of CD25 low and the 5\% highest population for CD25. Sorting purity controls are shown. Cells were then expanded for 14 days and used for experimentation between day 15 and day 17 . (B) Example of intracellular staining for FoxP3 on day 15, and (C) the quantification of percent FOXP3 ${ }^{+}$cells on day 15 of culture. (D-I) BSLBs can also be tailored for the study of CART cell $\mathrm{tSV}$. Data shown in A-E represent means +/- SEM from two independent experiments evaluating the transfer of the chimeric antigen receptor (CAR) (D), the TCR coreceptor $\mathrm{CD} 8(\mathrm{E}), \mathrm{CD} 63(\mathrm{~F})$, Perforin $(\mathrm{G})$, and as its comparison to $\mathrm{TCR} \alpha \beta$ at the tested densities (H). In D-H, Normality was determined using Shapiro-Wilk test and statistical significance was determined by Multiple t-test. $* \mathrm{p} \leq 0.05, \quad * * \mathrm{p} \leq 0.005, \quad * * * \mathrm{p} \leq 0.0005$, $* * * * \mathrm{p} \leq 0.0001$ and $\mathrm{ns}=$ not significant. (I) Representative pseudocolor plots showing the gating strategy for identifying single BSLBs and single CART. (D-G) Representative halfoverlaid histograms are shown to the right. The differences in synaptic transfer at comparable $\alpha-\mathrm{CD} 3 \varepsilon$ and HLA-A2: NY-ESO-1 densities $\left(140\right.$ molec./ $\left.\mu \mathrm{m}^{2}\right)$. tSV transfer instigated by either the CAR antigen (top panels; teal BSLBs), or the $\alpha-C D 3 \varepsilon$ Fab (bottom panels, violet BSLBs) are shown for each marker. Cells are shown in dark blue (no antigen/ $\alpha-C D 3$ ), and light blue (co-cultured with either HLA-A2: NY-ESO-1 or $\alpha$-CD3e). Light grey BSLBs represent those loaded with no antigen. (J-K) Increased synaptic transfer of CD39 (J) and CD73 (K) to BSLB associates with higher central clustering of CD39 and CD73 in Treg synapses imaged by TIRFM. Left panels: TIRFM images comparing TH (top) and Treg 
(bottom) showing the distribution of the ectonucleotidases (A) CD39 and (B) CD73 in mature ISs. Middle panels show background subtracted mean fluorescence intensities (corrected MFI) for CD39 and CD73 in the contacts of TH and Treg cells stimulated on planar glass SLB containing 200 molec./ $\mu \mathrm{m}^{2}$ of ICAM1, 20 molec./ $\mu \mathrm{m}^{2}$ of CD40 and $30 \mathrm{molec} . / \mu \mathrm{m}^{2}$ of $\alpha-C D 3 \varepsilon$ Fab (clone UCHT-1). SIRC: surface interference reflection contrast microscopy. Cell surface glycans are labelled with Wheat Germ Agglutinin (WGA). White arrowheads show released $\mathrm{WGA}^{+} \mathrm{CD}^{+}$vesicles (diffraction limited). Scale bar $=5 \mu \mathrm{m}$. Two independent experiments are shown. Points represent individual synapses. Right panels: transfer behavior to BSLB relates to increased surface expression of ectonucleotidases in Treg cells (blue) compared to TH (green). Normality was determined using Shapiro-Wilk test and statistical significance was determined by two-tailed Mann-Whitney unpaired test. $* \mathrm{p} \leq 0.05, * * \mathrm{p} \leq 0.005, * * * \mathrm{p} \leq 0.0005, * * * * \mathrm{p} \leq 0.0001$ and $\mathrm{ns}=$ not significant.

Supplementary Figure 5; related to Fig. 4. (A) Surface protein expression for TH from 9 different donors edited using CRISPR/Cas9 targeting CD4, CD81, ADAM10, BST2, and TSG101. (B) Representative half-overlaid histograms and bi-parametric contour plots showing the surface expression levels of edited proteins (headings) and their relationship with the expression of CD40L on cells (Y-axis). (C) Relationship between CD40L expression and $\mathrm{CD} 3 \xi$ expression CD4- (teal) and ADAM10-edited cells (orange). (D) TSG101 downregulation resulting from CRISPR/Cas9-genome editing shown normalized to beta-actin (left panel). (E-J) Dynamics of tSV transfer between different CRISPR/Cas9 edited cells and the resulting downregulation of the markers on the cell surface. Non-linear, three-parameter regression analyses (F-test) comparing $\mathrm{TCR} \alpha \beta^{+}$and $\mathrm{CD} 40 \mathrm{~L}^{+} \mathrm{tSV}$ transfer among CRISPRCas9 knockdowns and in response to increasing densities of agonistic $\alpha$-CD3e-Fab. (E) $\mathrm{T}_{\max }$ for $\mathrm{TCR}^{+}$and $\mathrm{CD}_{40 \mathrm{~L}}{ }^{+} \mathrm{tSV}$ transfer to BSLB among different CRISPR/Cas9-edited cells. 
Data represent means normalised to $C D 4$-edited controls. (F) $\alpha-\mathrm{CD} 3 \varepsilon$ Fab $\mathrm{EC}_{50}$ for $\mathrm{TCR}^{+}$and $\mathrm{CD}_{40 \mathrm{~L}^{+}} \mathrm{tSV}$ transfer to BSLB among different CRISPR/Cas9-edited cells. Data represent means normalised to $C D 4$-edited controls. (G) $\mathrm{TCR} \alpha \beta^{+}$tSV (as $\mathrm{TCR} \alpha \beta^{+}$molec./BSLB) transfer to BSLB at increasing $\alpha-C D 3 \varepsilon$ Fab densities, and $(\mathrm{H})$ its resulting downregulation on the surface of $\mathrm{T}$ cells $(\mathrm{H})$. (I) $\mathrm{CD}_{40 \mathrm{~L}^{+}} \mathrm{tSV}$ (as CD40L ${ }^{+}$molec./BSLB) transfer to BSLB at increasing $\alpha-\mathrm{CD} 3 \varepsilon$ Fab densities, and $(\mathrm{J})$ its resulting upregulation on the surface of $\mathrm{T}$ cells following stimulation with $\alpha-\mathrm{CD} 3 \varepsilon-\mathrm{Fab}$.

Supplementary Figure 6; related to Fig. 5. (A) Sorting of BSLB after conjugate dissociation for the sequencing of tSVs small RNA content. Upper panel: Pseudocolor plots showing the gating strategy for sorting single BSLB for the downstream sequencing of small RNAs. Shown are post-sorting controls. Right overlaid histograms showing TCR $\alpha \beta$ on BSLB loaded with $\alpha-C D 3 \varepsilon$ Fab (violet) versus null controls (grey) after sorting from T cell: BSLB co-cultures. Bottom panel: post-sort control showing the enrichment of single cells. (B) KEGG GO pathway enrichment analyses reveals unique miR to both T-cell tSV and EVs and sharing association with common biological processes. Heatmaps detailing miR species and KEGG GO pathways functionally enriched in TH tSV, TH EVs, CTL tSVs and CTL EVs; Heatmap values represent $\log 10$ p-values. (C-F) Bubble plots depicting enriched target pathways associated with miR identified in TH tSV (C), TH EVs (D), CTL tSVs (E), and CTL EVs (F). Circles represent different GO terms, and circle size represents the number of targets associated with the given GO category. The overall colour represents the cluster (a broader category) encompassing the enriched GO categories. $(\mathrm{G}-\mathrm{H})$ Number of miR targets across different GO pathways. CTL tSV and EVs $(\mathrm{G})$, and TH tSVs and EVs $(\mathrm{H})$ are compared. Shared miR targets per GO category are shown in grey, unique targets for tSVs are shown in magenta and unique targets for EVs miR are shown in green. 


\section{Supplementary Figure 7; related to Fig. 5. SVs are enriched in RBPs, which localise as} discrete puncta in the synaptic cleft of mature TH ISs. (A) LC-MS/MS analyses of enriched proteins found in eluted tSVs from a-CD3 coated BSLB and null BSLB containing only ICAM1 and CD40. (B-C) differentially expressed proteins in tSV were then used for the differential expression analyses of tSV with EVs from TH (B) and CTL (C). (D-G) Enriched proteins in $\mathrm{tSV}$ and EVs were identified by averaged-based normalisation of peptide intensities ( $\log 10)$. (D) tSV and EVs showing the differential enrichment of contaminating proteins. (E) . (F) GSEA using PANTHER [79] revealed a higher enrichment of nucleic-acid binding proteins in the LC-MS/MS of tSV (magenta) derived from TH (left) and CTL (middle left) as compared with syngeneic EVs (green) of TH (middle right) and CTLs (right). Values represent the fold enrichment of identified proteins per GO term. (H) Venn-Diagram showing the co-identification of RBPs in LC-MS/MS datasets generated by two different isolation methods, namely tSV elution and sorting of BSLB. (I) TIRFM imaging of the RBP YBX1 in the SLB-contacting pole of T cell IS after 20 minutes of interaction with SLBs. YBX1 was enriched in TH tSV as defined by analyses in D and in new and previously published[5] proteomics from isolated ectosomes. $\mathrm{T}$ cells were fixed, permeabilised, and stained with monoclonal antibodies against vimentin for the identification of the pSMAC (yellow) and the cSMAC (dark central area). White arrows indicate the localisation of YBX1 in vesicular tracks of cells resuming migration and in the cSMAC -synaptic cleft. (J) as in I, T cells were stained against SF3B3[5]. (K) A rabbit monoclonal IgG isotype control was used as a control. (L-M) TH cells were pre-stained with BODIPYTM TR Ceramide and SYTO ${ }^{\mathrm{TM}}$ RNASelect ${ }^{\mathrm{TM}}$ to track the polarisation and localisation of ceramide and RNA enriched structures in the synaptic cleft. (L) Examples of some T cell kinapses showing the release of ceramide ${ }^{+}$and $\mathrm{RNA}^{+}$vesicles in the tracks of cells resuming migration (yellow arrows). (M) 
Left: Example of a TH synapse showing localisation of ceramide ${ }^{+}$and $\mathrm{RNA}^{+}$puncta in transit to the cSMAC. Right: We used the super-resolution provided by eTIRF-SIM to visualise the arrangement of $\mathrm{RNA}^{+}$puncta at 5 min of synapse assembly. Most puncta associate to ceramide enriched ER-like structures lining the cSMAC (synaptic cleft, yellow arrows), suggesting the rapid polarisation of RNA transport machineries to the boundary of the synaptic cleft. Scale bars $=5 \mu \mathrm{m}$. Imaging representative of at least three independent experiments and four different donors.

\section{ACKNOWLEDGEMENTS}

We are grateful to our laboratory members and the Kennedy Institute of Rheumatology community for constructive scientific discussions, especially to James Felce, Jonathan Webber, Štefan Balint, Alexander Mørch and Kristina Correa. We thank the technical support of Heather Rada, Kellie Johnson and Ekaterina Zvezdova (the latter two from BioLegend). We thank Professor Catarina E. Hioe for kindly providing the HIV-1 gp120 protein. We would also like to thank all the anonymised blood donors who contributed to our study. This work was funded by Wellcome Trust Principal Research Fellowship 100262Z/12/Z, the ERC Advanced Grant (SYNECT AdG 670930), and the Kennedy Trust for Rheumatology Research (KTRR) (all three to M.L.D.). P.F.C.D was supported by EMBO Long-Term Fellowship (ALTF 1420-2015, in conjunction with the European Commission (LTFCOFUND2013, GA-2013-609409) and Marie Sklodowska-Curie Actions) and OxfordBristol Myers Squibb Fellowship. A.K. was supported by H2020 and the Research Council of Norway (in conjunction with Marie Sklodowska-Curie Actions 275466; to A.K.). M.F. and H.C.Y. thank the Wellcome Trust (212343/Z/18/Z) and EPSRC (EP/S004459/1). The eTIRFSIM platform was built in collaboration with Micron (www.micronoxford.com), an Oxfordwide advanced microscopy technology consortium supported by Wellcome Strategic Awards 
(091911 and 107457), and with additional funds from an MRC/EPSRC/BBSRC nextgeneration imaging award and the Kennedy Trust for Rheumatology Research through the Kennedy Institute Cell Dynamics Platform. We acknowledge the generous support of the Kennedy Trust for Rheumatology Research, IDRM and Carl Zeiss GMBH for the Airyscan LSM 980 confocal microscope used in this research. Y.P., T.D. and R.F. were supported by the Chinese Academy of Medical Sciences (CAMS) Innovation Fund for Medical Sciences (CIFMS), China (grant number: 2018-I2M-2-002) and UK Medical Research Council (MRC); E.S. was supported by Newton-Katip Celebi Institutional Links grant (352333122) and SciLifeLab fellowship (to E.S.). F.S-M. was supported by grants SAF2017-82886-R from the Spanish Ministry of Economy and Competitiveness (MINECO), and "La Caixa" Banking Foundation (HR17-00016). We thank the NIH Tetramer Core Facility for the synthesis of the HLA-DRB1*09:01 monomers used in this study. We thank the Oxford Genomics Centre at the Wellcome Centre for Human Genetics (funded by Wellcome Trust grant reference $203141 / \mathrm{Z} / 16 / \mathrm{Z}$ ) for the generation and initial processing of the sequencing data. Finally, we thank the MS laboratory at the Target Discovery Institute NDM (Oxford) led by Benedikt M. Kessler. Pablo F. Céspedes is also known as Pablo F. Céspedes-Donoso (https://orcid.org/0000-0002-1641-4107). 


\section{MATERIAL AND METHODS}

\section{Ethics}

Different $\mathrm{T}$ cell populations were isolated from leukapheresis reduction system (LRS) chambers from de-identified, non-clinical healthy donors. The non-clinical issue division of the National Health Service and the Inter-Divisional Research Ethics Committee of the University of Oxford approved the use of LRS chambers (REC 11/H0711/7 and R51997/RE001).

\section{Isolation and expansion of human $\mathrm{CD4}^{+}, \mathrm{CD8}^{+}$and Treg cells from peripheral blood.}

Briefly, human $\mathrm{T}$ cells were isolated from leukoreduction system (LRS)-concentrated peripheral blood by negative immunodensity selection using either $\mathrm{CD}^{+}, \mathrm{CD}^{+}$or $\mathrm{CD} 4^{+} \mathrm{CD} 127^{\text {low }}$ (RosetteSep ${ }^{\mathrm{TM}}$, StemCell ${ }^{\mathrm{TM}}$ Technologies, \#15022, \#15023, and \#15361). Enriched $\mathrm{CD} 4^{+} \mathrm{CD} 127^{\text {low }}$ cells were immediately used for fluorescence-activated cell sorting of regulatory T cells (Treg) based on low CD127 fluorescence and high CD25 fluorescence. Briefly, Tregs were sorted from enriched $\mathrm{CD} 4^{+} \mathrm{CD} 127^{\text {neg }}$ peripheral blood cells using a FACSAria III cell sorter. A nested gating strategy was used in which $\mathrm{CD} 4^{+} \mathrm{CD} 127^{\text {neg }}$ $\mathrm{CD} 25^{\text {high }}$ cells were gated such that only the brightest $5 \%$ of $\mathrm{CD} 25^{\text {high }}$ cells were sorted. Recovered cells were stimulated with Human T-activator CD3/CD28 Dynabeads (ThermoFisher) at a bead to cell ratio of $3: 1$ and $1,000 \mathrm{U} / \mathrm{mL}$ of recombinant human IL-2 (Peprotech). Fresh IL-2 containing media was added every $48 \mathrm{~h}$ and Human T-activator CD3/CD28 Dynabeads were added again at day 7 and removed at day 15 of expansion. This protocol allowed us the enhanced recovery of FoxP3 ${ }^{+} \mathrm{T}$ cells by day 15 of expansion (see Fig. S4A-C). On the other hand, conventional $\mathrm{CD}^{+}$and $\mathrm{CD} 8^{+} \mathrm{T}$ cells were expanded using a bead to cell ratio of 1:1 and $100 \mathrm{U} / \mathrm{mL}$ of IL-2. After 3 days of activation, Human T-activator CD3/CD28 Dynabeads were removed and the conventional $\mathrm{CD}^{+}$and $\mathrm{CD} 8^{+}$cells kept in 
complete RPMI media supplemented with $100 \mathrm{U} / \mathrm{mL}$ of IL-2. All BSLB synaptic transfer experiments were performed after preconditioning of T cells to IL-2-depleted complete RPMI 1640 media containing $10 \%$ of either heat-inactivated AB human serum (Treg \& controls) or foetal bovine serum, and $100 \mu \mathrm{M}$ non-essential amino acids, $10 \mathrm{mM}$ HEPES, $2 \mathrm{mM} \mathrm{L}-$ glutamine, $1 \mathrm{mM}$ sodium pyruvate, $100 \mathrm{U} / \mathrm{ml}$ of penicillin and $100 \mu \mathrm{g} / \mathrm{mL}$ of streptomycin).

\section{Culture of $\mathrm{CD}^{+} \mathrm{T}$ cell clones}

The HLA-DRB1*09:01-restricted $\mathrm{T}$ cell clones 16, 35 and 40 (all specific against the influenza $\mathrm{H} 3 \mathrm{HA}_{338-355}$ peptide: NVPEKQTRGIFGAIAGFI) were expanded using at a ratio of 1 clone: 2 feeder cells (irradiated, pooled PBMCs from 2-3 healthy donors) at a total cell concentration of $3 \times 10^{6}$ cells/mL in RPMI 1640 supplemented with $10 \%$ heat-inactivated AB human serum and $30 \mu \mathrm{g} / \mathrm{ml}$ of PHA for three days. Then, $100 \mathrm{U} / \mathrm{ml}$ of recombinant human IL-2 were added to fresh media, which was replaced every 2 days. Clones were used between days 8 and 12 of culture.

\section{Production of chimeric antigen receptor $(\mathrm{CAR})$ recombinant $\mathrm{CD8}^{+} \mathrm{T}$ cells}

The CAR constructs that bind the NY-ESO-1 ${ }_{157-165}$ HLA-A2 complex were a kind gift from Cristoph Renner (Zurich, Switzerland) and described elsewhere [80, 81]. Briefly, the T1 CAR binds the C9V NY-ESO-1 APL with a $\mathrm{K}_{\mathrm{D}}$ of $\sim 2 \mathrm{nM}$. The CAR construct was packaged within third-generation self-inactivating lentiviral transfer vectors with the EF1 $\alpha$ promoter and the Woodchuck Hepatitis Virus Post-Transcriptional Regulatory Element. The CAR features a scFv binding domain, Ig domain spacers derived from human IgG1, the CD28 transmembrane domain and the CD3 $\zeta$ signalling tail. For lentiviral production, 293T cells (ATCC CRL-3216) were co-transfected with a mix of the VSV-G (370 ng), the lentiviral CAR (800 ng), and RSV-Rev and GAG (950 ng each) plasmids. The plasmid mix was 
prepared in DMEM (Merck, \#D6429) containing X-treme Gene HP DNA Transfection Reagent (Merck, \#6366546001) and after 20 min preincubation at RT, the mix was added to 293 cells in a dropwise manner. Twenty-four h after isolation and stimulation with Human T-activator CD3/CD28 Dynabeads, $\mathrm{CD} 8^{+} \mathrm{T}$ cells were transduced with freshly harvested and $0.45 \mu \mathrm{m}$-filtered supernatant containing $50 \mathrm{U} / \mathrm{mL}$ of recombinant human IL-2. Three days after transduction Dynabeads were removed and the cells kept in culture at a concentration of $1 \times 10^{6}$ cells/mL in IL-2 supplemented RPMI media (see above). Transduction efficiency was determined between days 8-10 of culture using a polyclonal goat anti-human IgG Fc PE (ThermoFisher Scientific, \#12-4998-82) and the synaptic transfer to BSLB addressed immediately.

\section{Bead Supported Lipid Bilayers (BSLB)}

Non-functionalized silica beads $(5.00 \pm 0.05 \mu \mathrm{m}$ diameter, Bangs Laboratories, Inc.) were washed extensively with PBS in $1.5 \mathrm{ml}$ conical microcentrifuge tubes. BSLBs were formed by incubation with mixtures of liposomes to generate a final lipid composition of $0.2 \mathrm{~mol} \%$ Atto-DOPE; $12.5 \mathrm{~mol} \%$ DOGS-NTA in DOPC at a total lipid concentration of $0.4 \mathrm{mM}$. In this work, we used DOPE lipids conjugated with either Atto 390, 488, 565 or 647 . The resultant BSLB were washed with $1 \%$ human serum albumin (HSA)-supplemented HEPESbuffered saline (HBS), referred herein as HBS/HSA buffer. To saturate NTA sites, BLSB were then blocked with $5 \%$ casein $100 \mu \mathrm{M} \mathrm{NiSO}_{4}$ for 20 minutes. After two washes, BSLB were loaded with concentrations of His-tagged proteins required to achieve the indicated molecular densities (in range of 1-100 nM; please refer to each Figure legend). Excess proteins were removed by washing with HBS/HSA after 30 minutes. T cells $\left(2.5 \times 10^{5} /\right.$ well $)$ were incubated with BSLB at 1:1 ratio in either U-bottomed or V-bottomed 96 well plate (Corning) for $90 \mathrm{~min}$ at $37^{\circ} \mathrm{C}$ in $100 \mu \mathrm{HBS} / \mathrm{HSA}$. For gentle dissociation of BSLB: cell 
conjugates, culture plates were gradually cooled down by incubation at RT for 15 min, followed by incubation on ice. After $45 \mathrm{~min}$, cells and BSLB were centrifuged at $300 \mathrm{~g}$ for 5 min prior to resuspension in ice-cold 5\% BSA in PBS pH 7.4. Single BSLB and cells were gently resuspended prior to staining for flow cytometry analysis or sorting.

\section{Multicolour Flow Cytometry (FCM)}

Staining with fluorescent dye conjugated antibodies was performed immediately after dissociation of cells and BSLB conjugates. Staining was performed in ice-cold 5\% BSA in PBS pH $7.4\left(0.22 \mu \mathrm{m}\right.$-filtered) for a minimum of $30 \mathrm{~min}$ at ${ }^{+} 4^{\circ} \mathrm{C}$. Then, cells and BSLB were washed three times and acquired immediately using an LSRFortessa X-20 flow cytometer equipped with a High Throughput Sampler (HTS). For absolute quantification, we used Quantum Molecules of Equivalent Soluble Fluorescent dye (MESF) beads (see below), which were first acquired to set photomultiplier voltages to position all the calibration peaks within an optimal arbitrary fluorescence units' dynamic range (between $10^{1}$ and $2 \times 10^{5}$, and before compensation). Fluorescence spectral overlap compensation was then performed using single colour-labelled cells and BSLB, and unlabelled BSLB and cells. For markers displaying low surface expression levels unstained and single colour stained UltraComp eBeads (Thermo Fisher Scientific Inc.; \#01-2222-42) were used for the calculation of compensation matrixes. After application of the resulting compensation matrix, experimental specimens and Quantum MESF beads were acquired using the same instrument settings. In most experiments acquisition was set up such as a minimum of $5 \times 10^{4}$ single BSLBs were recorded. To reduce the time of acquisition of high throughput experiments a minimum of $1 \mathrm{x}$ $10^{4}$ single BSLBs were acquired per condition instead. All dye-conjugated antibodies described in this study are listed in Table S5. 


\section{Calibration of flow cytometry (FCM) data}

$\mathrm{T}$ cells and BSLB were analysed using antibodies with known fluorescent dye to Ab ratio (F/P) in parallel with the Quantum MESF beads (Bangs Laboratories, Inc. IN, USA), allowing the calculation of the absolute number of antibodies bound per T cell and per BSLB after subtraction of unspecific signals given by isotype control antibodies. We used MESF standard beads labelled with the Alexa Fluor ${ }^{\circledR}$ dyes 488 and 647 to estimate number of dye molecules from mean fluorescence intensities (corrected and geometric, cGMFI). Briefly, MESF beads provided 5 different populations of beads with increasing GMFI allowing the linear regression of corrected GMFI (cGMFI) over MESF. The resulting slope is then used for the interpolation of total fluorochromes bound to either BSLB or cells from cGFMI values. Since we also used antibodies with known fluorochromes per protein $(\mathrm{F} / \mathrm{P})$, we then estimated the number of bound antibodies (and hence molecules) per BSLB by dividing the estimated fluorochrome number by the detection antibody F/P value (Number of molec./event $=$ Fluorescent molec $\left._{\text {event }} /(\mathrm{F} / \mathrm{P})_{\mathrm{Ab}}\right)$.

\section{Trans-synaptic vesicle elution from BSLBs}

Cells and BSLBs were incubated at a 1:1 ratio for 90 min at $37{ }^{\circ} \mathrm{C}$ and $5 \% \mathrm{CO}_{2}$ in Phenol Red-free FBS-free RPMI 1640 supplemented with $100 \mu \mathrm{M}$ non-essential amino acids, $2 \mathrm{mM}$ L-glutamine, $1 \mathrm{mM}$ sodium pyruvate, $100 \mathrm{U} / \mathrm{ml}$ of penicillin and $100 \mu \mathrm{g} / \mathrm{mL}$ of streptomycin. Cultures needed to be scaled up and therefore a $\mathrm{CO}_{2}$ incubator and supplemented FBS-free, Phenol Red-free RPMI 1640 was used instead of $1 \%$ HSA/HBS. Cells and BSLBs were collected at different time points of the elution protocol to control with FCM both the transfer of material from $\mathrm{T}$ cells to BSLB, and the release of $\mathrm{tSV}$ from BSLB upon addition of EDTA for the chelation of Ni. Briefly, elution was performed as follows; first conjugates were cooled down $15 \mathrm{~min}$ at RT and then $40 \mathrm{~min}$ on ice to gentle separate cells and BSLB. Then, 
conjugate suspensions were resuspended by adding 3 volumes of ice-cold PBS pH 7.4 and centrifuged at $100 \mathrm{~g}$ for $1 \mathrm{~min}$ and ${ }^{+} 4^{\circ} \mathrm{C}$ to enrich for BSLB. After two washes with ice cold PBS pH 7.4, BSLB were resuspended in ice-cold $50 \mathrm{mM}$ EDTA in PBS pH 7.4 for $2 \mathrm{~h}$. BSLB-free supernatants were then centrifuged at ${ }^{+} 4^{\circ} \mathrm{C}$ in sequential steps at $300 \mathrm{~g}$ for 5 min (twice), then at 2,000 $\mathrm{g}$ for $10 \mathrm{~min}$ and $10,000 \mathrm{~g}$ for $10 \mathrm{~min}$. Finally, recovered supernatants were centrifuged at $120,000 \mathrm{~g}$ for $4 \mathrm{~h}$ at ${ }^{+} 4^{\circ} \mathrm{C}$ and the pellets washed once more with PBS pH 7.4 and kept at ${ }^{+} 4{ }^{\circ} \mathrm{C}$ or frozen at $-80{ }^{\circ} \mathrm{C}$ until analyses by either NTA, NanoFCM or immunoblotting. Steadily released EVs were isolated by the same differential centrifugation procedure indicated above. To minimize serum and debris contamination, $\mathrm{T}$ cells were cultured in OptiMEM-I supplemented with $100 \mathrm{U} / \mathrm{mL}$ of IL-2, $100 \mu \mathrm{M}$ non-essential amino acids, $2 \mathrm{mM}$ L-glutamine, $1 \mathrm{mM}$ sodium pyruvate, $100 \mathrm{U} / \mathrm{ml}$ of penicillin and $100 \mu \mathrm{g} / \mathrm{mL}$ of streptomycin for no longer than $48 \mathrm{~h}$. All samples isolated by UC were resuspended in 0.22 $\mu \mathrm{m}$-filtered PBS pH 7.4 to reduce background signals in downstream particle measurement analyses with either nanoFCM or Nanoparticle Tracking Analysis (NTA).

\section{TEM}

The negative staining of thinly spread vesicle preparations for transmission electron microscopy (TEM) was performed as described elsewhere [82]. Briefly, carbon support filmcoated $3 \mathrm{~mm}$ copper grids (300 mesh) were plasma treated for $20 \mathrm{~s}$ using a Leica EM ACE200 Vacuum Coater. Then, $10 \mu \mathrm{L}$ of isolated vesicle populations were deposited on and incubated at RT for 5 min, followed by removal of excess sample with a Whatman $\mathrm{N}^{\mathrm{o}} 1$ paper and staining with $2 \%$ uranyl acetate for $10 \mathrm{~s}$ at RT. After removal of excess uranyl acetate the samples were dried for $10 \mathrm{~min}$ and analysed using a Tecnai $12 \mathrm{TEM}$ at $120 \mathrm{kV}$ using a Gatan OneView CMOS camera. A final magnification of 29,000x was used for imaging of isolated vesicle populations. 


\section{NTA}

Eluted tSV and purified EV preparations were resuspended in $0.22 \mu \mathrm{m}$-filtered PBS pH 7.4 in a 1:100 dilution and kept on ice for Nanoparticle Tracking Analysis (NTA). The instrument used for NTA was Nanosight NS300 (Malvern Instruments Ltd) set on light scattering mode and instrument sensitivity of 15 . Measurements were taken with the aid of a syringe pump to improve reproducibility. Three sequential recordings of 60 seconds each were obtained per sample and NTA 3.2 software was used to process and average the three recordings to determine the mean size.

\section{Nano Flow Cytometry}

Flow NanoAnalyzer model type N30E (NanoFCM Inc., Xiamen, China) that allows single ex osomes detection was used to determine the size distribution and granular concentration of $\mathrm{E}$ Vs. The Nano-flow cytometry analysis was performed using the Flow NanoAnalyzer (NanoFCM Co., LTD) according to manufacturer's instructions. The Silica Nanospheres Cocktail (S16M-Exo, NanoFCM) was employed as the size standard to construct a calibration curve to allow the conversion of side scatter intensities to particle size. A concentration standard (200nm PS QC beads, NanoFCM) was used to measure particle concentration. The laser used was a $488 \mathrm{~nm}$ laser at $25 / 40 \mathrm{~mW}, 10 \%$ ss decay. Lens filters equipped were 525/40 (AF488) and 580/40 (PE). Before staining samples were acquired to determine particle concentration such that a total of $10^{8}$ vesicles of either tSV or EVs were labelled per condition. Before use, fluorochrome-conjugated antibodies were spun down at $10,000 \mathrm{~g}$ for $10 \mathrm{~min}$ at $+4^{\circ} \mathrm{C}$. Isotype control antibodies were used at the same effective concentrations (ranging from 0.2 to $5 \mu \mathrm{g} / \mathrm{mL}$ ) and incubation times. Staining antibodies were conjugated to AF488 and AF647. Stainings were performed for 30 min on ice. Samples were 
washed and centrifuged for $1 \mathrm{~h}$ at $100,000 \mathrm{~g}$ and $+4^{\circ} \mathrm{C}$. Labelled vesicles were then resuspended in $50 \mu \mathrm{L}$ of PBS. Buffer alone (PBS), unstained vesicle, isotype controls and autothresholding were used to define positivity.

\section{Planar Supported Lipid Bilayers (PSLB)}

Liposome mixtures were injected into flow chambers formed by sealing acid piranha and plasma-cleaned glass coverslips to adhesive backed plastic manifolds with 6 flow channels (StickySlide VI 0.4; Ibidi). After 30 minutes the channels were flushed with HBS-HSA without introducing air bubbles to remove excess Liposomes. After blocking for 20 min with $5 \%$ casein supplemented with $100 \mu \mathrm{M} \mathrm{NiCl}_{2}$, to saturate NTA sites, followed by washing and then His-tagged proteins were incubated on bilayers for additional $30 \mathrm{~min}$. Protein concentrations required to achieve desired densities on bilayers were calculated from calibration curves constructed from FCM measurements on BSLB and analysed alongside MESF beads (MESF; Quantum ${ }^{\mathrm{TM}}$ Bangs Laboratories Inc.). Bilayers were continuous liquid disordered phase as determined by fluorescence recovery after photobleaching with a $10 \mu \mathrm{m}$ bleach spot on an FV1200 confocal microscope (Olympus).

\section{Immunological Synapse formation on glass-SLB}

Prior to immunological synapse imaging, primary $\mathrm{T}$ cell blasts were washed twice and resuspended in prewarmed HBS/HSA buffer to a final concentration of $5 \times 10^{6}$ cells $/ \mathrm{mL}$. Then, $5 \times 10^{5} \mathrm{~T}$ cells were stimulated for $20 \mathrm{~min}$ at $37^{\circ} \mathrm{C}$ and $5 \% \mathrm{CO}_{2}$ over PSLB containing

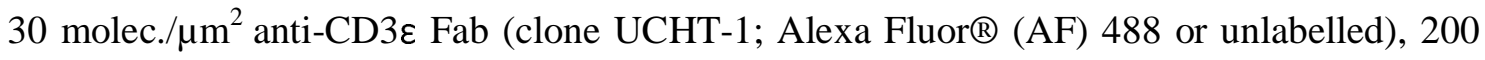
molec./ $\mu \mathrm{m}^{2}$ of ICAM1 AF405, and $100 \mathrm{molec} . / \mu \mathrm{m}^{2}$ of CD40 (AF488 or unlabelled). Then, cells were stained with $1 \mu \mathrm{g} / \mathrm{mL}$ of wheat germ agglutinin (WGA) conjugated to CF568 (Biotium; \#29077-1) and $1 \mu \mathrm{g} / \mathrm{mL}$ anti-CD154 (CD40L) clone 24-31 AF647 for $15 \mathrm{~min}$ at 
RT and in the dark. Cells were washed three times with 5\% BSA $20 \mathrm{mM}$ HEPES $2 \mathrm{mM}$ $\mathrm{MgCl}_{2}$ in PBS and fixed with pre-warmed 4\% electron microscopy grade formaldehyde in PBS pH 7.4 containing $2 \mathrm{mM} \mathrm{MgCl}_{2}$ for $10 \mathrm{~min}$ at $\mathrm{RT}$ and in the dark. After two washes, cells were kept in HBS/HSA until imaging by TIRFM. For intracellular staining, cells were permeabilized for 3 min with $0.1 \%$ Triton X-100 in PBS, washed and blocked for 60 min with 5\% BSA in PBS before staining with primary antibodies $(1 \mu \mathrm{g} / \mathrm{mL}$ for $1 \mathrm{~h}$ at RT). Primary antibodies included Rabbit anti-human TSG101 clone EPR7130B AF647 (\#ab207664), anti-human YB1 (YBX1) clone EPR22682-2 (\#ab255606), anti-human SF3B3 clone EPR18440 (\#ab209402), and Rabbit Isotype control EPR25A AF647 (\#ab199093). After three washes, cells were blocked with $0.22 \mu \mathrm{m}$-filtered 5\% Donkey Serum for $1 \mathrm{~h}$ at RT before staining for $1 \mathrm{~h}$ with AF647 AffiniPure F(ab') $\square$ Fragment Donkey Anti-Rabbit IgG (H+L) (Jackson ImmunoResearch, \#711-606-152). After four washes, cells were washed and then stained with anti-Vimentin clone EPR3776 AF555 (\#ab203428). Cells were washed four times before acquisition.

\section{TIRFM}

TIRFM was performed on an Olympus IX83 inverted microscope equipped with a 4-line (405 nm, $488 \mathrm{~nm}, 561 \mathrm{~nm}$, and $640 \mathrm{~nm}$ laser) illumination system. The system was fitted with an Olympus UApON 150x 1.45 numerical aperture objective, and a Photometrics Evolve delta EMCCD camera to provide Nyquist sampling. Quantification of fluorescence intensity was performed with Fiji/ImageJ (National Institute of Health) and MATLAB R2019b. A batch measure macro was used to automatically segment cell:SLB contacts based on internal reflection microscopy followed by both background subtraction and the measure of fluorescence across different channels. 


\section{Airyscan microscopy}

Airyscan imaging of $\mathrm{T}$ cell and SLB was performed on a Zeiss Axio Observer.Z1 LSM 980 confocal laser-scanning microscope equipped with an Airyscan 2 module (Zeiss, Oberkochen, Germany) consisting of 32 concentrically arranged GaAsP PMT detectors and 2 MA-PMT channels. The acquisition was performed using the Airyscan super-resolution (SR) and best signal Smart Setup and a C Plan-Apochromat 63x/1.4 NA Oil DIC magnification objective. Illumination was provided by a Solid-State Light Source Colibri 7 LED lamp and by Diode lasers at $639 \mathrm{~nm}, 594 \mathrm{~nm}$, and $488 \mathrm{~nm}$ with $0.4 \%$ laser power and $850 \mathrm{~V}$ detector gain for all channels. The imaging field was defined using a $6.0 \mathrm{X}$ scan zoom (crop area) and a Z-coverage spanning the totality of the synaptic cleft as defined by WGA staining. The final acquisition settings included a sequential acquisition in the order $647 / 594 / 488$, a frame size of 528 x 528 px, a pixel time of $7.95 \mu \mathrm{s}$, a pixel size of $0.043 \mathrm{x}$ $0.043 \times 0.16 \mu \mathrm{m}$, and a doubled pixel sampling with bidirectional mean intensity averaging of acquisition lines. Analyses were performed using the ZEN 3.2 system blue edition (Carl Zeiss Microscopy GmbH), and Fiji v2.1.0/1.53c (build 5f23140693)[83].

\section{eTIRF-SIM}

A custom-built eTIRF-SIM microscope system was used and detailed elsewhere[84]. Structured illumination was obtained via a grating pattern generated by a ferroelectric spatial light modulator (SLM, Forth Dimension Displays, QXGA3DM). After, the first diffraction orders are selected by the mask and sent to the Olympus IX83 microscope head. The distance between diffraction orders were tuned by SLM settings ultimately defining the illumination angle and therefore the TIRF depth. Excitation wavelengths of $488 \mathrm{~nm}, 560 \mathrm{~nm}$, and $640 \mathrm{~nm}$ were used (MPB communications Inc., 500mW, 2RU-VFL-P-500-488-B1R, 2RU-VFL-P500-560-B1R). Sample illumination was carried out using a high-NA TIRF objective 
(Olympus Plan-Apochromat 100X 1.49NA). The emitted fluorescence was collected by the same objective and sent onto sCMOS cameras recording the raw data (Hamamatsu, Orca Flash 4.0 v2 sCMOS). The excitation numerical aperture (NA) was adjusted for each wavelength by changing the period of the grating pattern at the SLM, which allows controlling the TIRF angle and, therefore, the penetration depth of the evanescent wave. To achieve TIRF-SIM illumination at the interface between the cells and SLB, both excitation lights were sent with an incident NA ranging from 1.38 to 1.41. Prior to experiment acquisition alignment was perform by imaging $100 \mathrm{~nm}$ Tetraspeck fluorescent beads in all three excitation colours. Then, using Fiji plugin MultiStackReg - the beads images were used to adjust images and compensate for chromatic aberrations. A total of 9 raw images were acquired per frame and for a single excitation wavelength before switching to the next wavelength. Then, raw images were processed and reconstructed into SIM images by custom made software or ImageJ fairSIM plugin. All experiments were performed at physiological conditions using a micro-incubator (H301, Okolabs, Italy) at $37{ }^{\circ} \mathrm{C}$ and $5 \% \mathrm{CO}_{2}$. For each frame, we used an acquisition time between 20 and $300 \mathrm{~ms}$ depending on the fluorescence signal levels and 2 colours, 18 frames total) every $0.4-5$ s. One colour super-resolved image was reconstructed from 9 raw image frames (3 angles and 3 phases) using a reconstruction method described previously $[85,86]$.

\section{CRISPR/Cas9 genome editing}

After $48 \mathrm{~h}$ of stimulation with Human T-activator CD3/CD28 Dynabeads (ThermoFisher) $\mathrm{CD}^{+}$lymphoblasts were recovered for transfection of CRISPR/Cas9 nucleoprotein complexes. Briefly, tracer RNA and crRNAs were mixed at equimolar concentrations (200 $\mu \mathrm{M})$ and incubated at $95^{\circ} \mathrm{C}$ for $15 \mathrm{~min}$. After cooling the gRNA mix down to RT, the Cas9 enzyme was added at final $20 \mu \mathrm{M}$ (IDT, \#1081061). After $15 \mathrm{~min}$ incubation at $37^{\circ} \mathrm{C}$, 
electroporation enhancer was added to CRISPR-Cas9 nucleoprotein complexes following manufacturer's guidelines, and immediately mixed with $1.5 \times 10^{6}$ cells. Cells were then transferred to a 2-mm cuvette (Bio-Rad) and electroporated at $300 \mathrm{~V}$ for $2 \mathrm{~ms}$ using an ECM830 Square Wave electroporator (BTX). Immediately after transfection, cells were recovered with pre-warmed, IL-2 supplemented RPMI 1640 media and expanded for another 6 days. Synaptic transfer experiments to BSLB or planar SLB were performed on day 7 or 8 of culture and protein expression controls were carried out in parallel. All crRNAs sequences are summarised in Table S6. All on- and off-target scores were optimised by the crRNA supplier (IDT).

\section{Western Blotting}

Whole cell lysates (WCL) were prepared by resuspending cell pellets in RIPA lysis and extraction buffer (Thermo Fisher Scientific, \#89901) containing a Protease/Phosphatase inhibitor cocktail (Cell Signaling Technology (CST); \#5872) to a final concentration of $2 \mathrm{x}$ $10^{7}$ cells $/ \mathrm{mL}$. After sonication at $+4^{\circ} \mathrm{C}(10$ cycles of $30 \mathrm{~s}$ on $/ 30 \mathrm{~s}$ off $)$, WCL were centrifuged at $10,000 \mathrm{~g}$ for $10 \mathrm{~min}$ at ${ }^{+} 4^{\circ} \mathrm{C}$, and the supernatants collected, mixed with loading solution and denatured at ${ }^{+} 95^{\circ} \mathrm{C}$ for 10 min. For immunoblotting of BSLB eluted material, after centrifugation at $120,000 \mathrm{~g}$ for $4 \mathrm{~h}$ at ${ }^{+} 4^{\circ} \mathrm{C}$, pellets were resuspended in RIPA lysis buffer containing a Protease/Phosphatase inhibitor cocktail, mixed with loading solution and denatured at ${ }^{+} 95^{\circ} \mathrm{C}$ for $10 \mathrm{~min}$. When indicated, lysed eluates and $\mathrm{EVs}$ from an equivalent number of originating cells were resolved to compare different vesicle populations. As positive control we used WCL equivalent to $2.25 \times 10^{5} \mathrm{CD}^{+}$lymphoblasts. Similarly, for CRISPR/Cas9 edited cells, WCL equivalent to $3 \times 10^{5}$ cells were used per lane. Samples were resolved using 4-15\% Mini-PROTEAN SDS-PAGE gel (Bio-Rad; \#4561084), transferred to $0.45 \mu \mathrm{m}$ nitrocellulose membranes (Bio-Rad, \#1620115), blocked and 
incubated with the following primary antibodies: rabbit anti-human CD40 Ligand clone D5J9Y (CST, \#15094), rabbit anti-GAPDH clone D16H11 (CST, \#5174), mouse anti- $\beta$-actin clone 8H10D10 (CST, \#3700), and rabbit anti-TSG101 clone EPR7130B (\#ab125011). Then, membranes were incubated with IRDye ${ }^{\circledR}$ 680RD donkey anti-mouse IgG (H+L; LI-COR, \#926-68072) and IRDye® 800CW donkey anti-rabbit IgG (H+L; LI-COR, \#925-32213) secondary antibodies following manufacturer guidelines. After four washes, membranes were imaged and analysed using the Odyssey® CLx Near-Infrared detection system equipped with the Image Studio ${ }^{\mathrm{TM}}$ Lite quantification software (LI-COR, Lincoln, NE).

\section{RNA sequencing and miR analyses}

Total cell RNA was extracted using the miRNeasy Tissue/Cells Advanced Mini Kit (Qiagen, \#217604). Purified RNA yields and quality were assessed via Agilent 2100 Bioanalyzer using the Agilent 6000 RNA Pico chips (Agilent Technologies, \# 5067-1513). Before library preparation the material was further quantified using RiboGreen (Invitrogen) on the FLUOstar OPTIMA plate reader (BMG Labtech) and the size profile and integrity analysed on the 2200 or 4200 TapeStation (Agilent, RNA ScreenTape). Input material was normalised to 200 ng or maximum mass for input volume prior to library preparation. Small RNA library preparation was completed using NEBNext Small RNA kit (NEB) following manufacturer's instructions and applying the low input protocol modifications. Libraries were amplified (15 cycles) on a Tetrad (Bio-Rad) using in-house unique dual indexing primers as described elsewhere [87]. Size selection was performed using Pippin Prep instrument (Sage Science) using the 3\% Agarose, dye free gel with internal standards (size selection: 120 to 230bp). Individual libraries were normalised using Qubit, and the size profile was analysed on the 2200 or 4200 TapeStation. $10 \mathrm{nM}$ libraries were denatured and further diluted prior to loading on the sequencer. Two runs of single end sequencing were performed; run one was 
performed in an Illumina HiSeq 2500 system (1x60) and run two was performed using Illumina NextSeq 500/550 v2.5 Kits (75 cycles). Quality control and processing of the raw sequencing data was performed using sRNAbench [88] and miRQC [89] which allowed the assessment of sequencing yield, quality, percentage of miR-mapping reads, read length distribution and relative abundance of fragments from other RNA species. Functional and biological pathway enrichment analyses were performed on annotated miR species shared between the two independent sequencing runs and enriched in each EV category (i.e., SV and sEV). MIENTURNET and the KEGG annotation database were used. Statistical analysis for functional and biological pathway enrichment analyses using MIENTURNET were carried out by calculating the $P$-value resulting from the hypergeometric test. Motif analyses of bulk, enriched miR sequences in each sample were performed using MEME (Multiple Em for Motif Elucidation 5.1.0) [42]. To dissect the miR-target gene interactome of our enriched miR we used miRNet 2.0 [90] by first mapping input miR to the miR interaction knowledgebase comprising annotations from miRbase, miTarBase and ExoCarta together with interactions with other miR, genes and transcription factors from TransmiR 2.0, ENCODE, JASPAR and ChEA. The output of the 'miRs' module of miRNet 2.0 provided the motif miR-target gene interactome enrichment analysis. We used FunSet [91] to graphically plot gene ontology (GO) enrichment analyses in 2D plots clustering GO terms based on semantic similarities and extracting representative terms for each cluster. The result of GO enrichment analysis using Funset was filtered by setting the FDR threshold to 0.05 (using the Benjamini-Hochberg procedure) and the $P$-values were calculated using the hypergeometric test.

\section{Mass spectrometry}


Samples isolated by differential centrifugation were concentrated using 100,000 NMWL centrifugal filters (Amicon $®$ Ultra, \#UFC510024, Merck Millipore Ltd.) and prepared in STrap $^{\mathrm{TM}}$ spin columns (\#C02-micro-80) following manufacturer recommendations. Briefly, samples were reduced with $5 \mu \mathrm{L}$ of $10 \mathrm{mM}$ TCEP and alkylated with $50 \mathrm{mM}$ of IAA for 30 min each, then acidified with $12 \%$ phosphoric acid 10:1 vol:vol, and then transferred to Strap columns. Then, samples were precipitated using 1:8 vol:vol dilution of each sample in 90\% methanol in $100 \mathrm{mM}$ TEAB. Samples were then washed three times with $90 \%$ methanol in $100 \mathrm{mM}$ TEAB. Samples were then resuspended in $50 \mu \mathrm{L}$ of $50 \mathrm{mM}$ TEAB and digested with trypsin (Promega, \#V1115) overnight at $37^{\circ} \mathrm{C}$. Peptides were eluted from the S-Trap by spinning for $1 \mathrm{~min}$ at $1,500 \mathrm{~g}$ with $80 \mu \mathrm{L}$ of $50 \mathrm{mM}$ ammonium bicarbonate, $80 \mu \mathrm{L} 0.1 \% \mathrm{FA}$ and finally $80 \mathrm{~mL}$ of $50 \%$ ACN $0.1 \%$ FA. The eluates were dried down in a vacuum centrifuge and resuspended in $2 \%$ ACN $0.1 \%$ TFA prior to off-line high-pH reversed-phase fractionation using RP-S cartridges pre-primed with $100 \mu \mathrm{L}$ ACN at $300 \mu \mathrm{L} / \mathrm{min}$ and equilibrated with $50 \mu \mathrm{L}$ of $2 \%$ ACN $0.1 \%$ TFA at $10 \mu \mathrm{L} / \mathrm{min}$. Samples were loaded at 5 $\mathrm{mL} / \mathrm{min}$ and divided into 8 fractions (elution steps), which were run individually. Elutions were performed with increasing concentrations of $90 \% \mathrm{ACN}, \mathrm{pH} 10$ in water, including final $5 \%, 10 \%, 12.5 \%, 15 \%, 20 \%, 22.5 \%, 25 \%$ and $50 \%$. Fractions were further dried down in a vacuum centrifuge and resuspended in loading buffer. For LC-MS/MS acquisition 50-80 ng peptides were loaded onto preconditioned Evotips containing $0.1 \%$ FA in water. Preconditioning was performed by pre-priming of isopropanol-soaked tips with $20 \mu \mathrm{L}$ of ACN $0.1 \% \mathrm{FA}$, following by centrifugation for $1 \mathrm{~min}$ at $700 \mathrm{~g}$, equilibration in water $0.1 \%$ FA and a final centrifugation for $1 \mathrm{~min}$ at $700 \mathrm{~g}$ ). Samples were run on a LC-MS system comprised of an Evosep One and Bruker timsTOF Pro. Peptides were separated on an $8 \mathrm{~cm}$ analytical C18 column (Evosep, $3 \mu \mathrm{m}$ beads, $100 \mu \mathrm{m}$ ID) using the pre-set 60 samples per day gradient on the Evosep One. Acquisition was done in PASEF mode (4 PASEF frames, 3 
cycles overlap, oTOF control v6.0.0.12) including an ion mobility window between 1/k0 start $=0.85 \mathrm{Vs} / \mathrm{cm} 2$ to $1 / \mathrm{k} 0$ end $=1.3 \mathrm{Vs} / \mathrm{cm}^{2}$, a ramp time of $100 \mathrm{~ms}$ with locked duty cycle and a mass range of $100-1,700 \mathrm{~m} / \mathrm{z}$. For proteomic analyses the raw files were searched against the reviewed Uniprot Homo sapiens database (retrieved 2,01,80,131) using MaxQuant version 1.6.10.43 [92] and its built-in contaminant database using tryptic specificity and allowing two missed cleavages.

\section{Statistical Analyses}

Normality tests were performed using either Shapiro-Wilk or Kolmogorov-Smirnov tests. Statistical significance was determined by multiple comparisons performed either by oneway analysis of variance (ANOVA) or Kruskal-Wallis (corrected by Holm-Sidak or Dunn's, respectively). Non-linear regressions using three or four parameters F-tests were also performed when indicated. All statistical tests were performed in GraphPad Prism v8 and are detailed in each figure legend. Means of significance are as follows: $* \mathrm{p}<0.05, * * \mathrm{p}<0.005$, $* * * \mathrm{p}<0.0005$, and $* * * * \mathrm{p}<0.0001$ 


\section{REFERENCES}

1. Gerard, A., et al., Secondary $T$ cell-T cell synaptic interactions drive the differentiation of protective CD8+ T cells. Nat Immunol, 2013. 14(4): p. 356-63.

2. $\quad$ Cespedes, P.F., et al., Model membrane systems to reconstitute immune cell signaling. FEBS J, 2021. 288(4): p. 1070-1090.

3. Mittelbrunn, M., et al., Unidirectional transfer of microRNA-loaded exosomes from $T$ cells to antigen-presenting cells. Nat Commun, 2011. 2: p. 282.

4. Choudhuri, K., et al., Polarized release of T-cell-receptor-enriched microvesicles at the immunological synapse. Nature, 2014. 507(7490): p. 118-23.

5. Saliba, D.G., et al., Composition and structure of synaptic ectosomes exporting antigen receptor linked to functional CD40 ligand from helper T cells. Elife, 2019. 8.

6. Balint, S., et al., Supramolecular attack particles are autonomous killing entities released from cytotoxic T cells. Science, 2020. 368(6493): p. 897-901.

7. Huang, J.F., et al., TCR-Mediated internalization of peptide-MHC complexes acquired by T cells. Science, 1999. 286(5441): p. 952-4.

8. Hudrisier, D., et al., Cutting edge: CTLs rapidly capture membrane fragments from target cells in a TCR signaling-dependent manner. J Immunol, 2001. 166(6): p. 36459.

9. Tabiasco, J., et al., Acquisition of viral receptor by NK cells through immunological synapse. J Immunol, 2003. 170(12): p. 5993-8.

10. Qureshi, O.S., et al., Trans-endocytosis of CD80 and CD86: a molecular basis for the cell-extrinsic function of CTLA-4. Science, 2011. 332(6029): p. 600-3.

11. Hou, T.Z., et al., A transendocytosis model of CTLA-4 function predicts its suppressive behavior on regulatory T cells. J Immunol, 2015. 194(5): p. 2148-59.

12. Jeppesen, D.K., et al., Reassessment of Exosome Composition. Cell, 2019. 177(2): p. 428-445 e18.

13. Chouaki-Benmansour, N., et al., Phosphoinositides regulate the TCR/CD3 complex membrane dynamics and activation. Sci Rep, 2018. 8(1): p. 4966.

14. Schneider, F., et al., Diffusion of lipids and GPI-anchored proteins in actin-free plasma membrane vesicles measured by STED-FCS. Mol Biol Cell, 2017. 28(11): p. 1507-1518.

15. Beckers, D., D. Urbancic, and E. Sezgin, Impact of Nanoscale Hindrances on the Relationship Between Lipid Packing and Diffusion in Model Membranes. J Phys Chem B, 2020.

16. Natkanski, E., et al., B cells use mechanical energy to discriminate antigen affinities. Science, 2013. 340(6140): p. 1587-90.

17. Wang, T., et al., Dynasore-induced potent ubiquitylation of the exon 19 deletion mutant of epidermal growth factor receptor suppresses cell growth and migration in non-small cell lung cancer. Int J Biochem Cell Biol, 2018. 105: p. 1-12.

18. Isaaz, S., et al., Serial killing by cytotoxic $T$ lymphocytes: $T$ cell receptor triggers degranulation, re-filling of the lytic granules and secretion of lytic proteins via a nongranule pathway. Eur J Immunol, 1995. 25(4): p. 1071-9.

19. Li, J.H., et al., The regulation of CD95 ligand expression and function in CTL. $\mathrm{J}$ Immunol, 1998. 161(8): p. 3943-9.

20. Sundquist, W.I., et al., Ubiquitin recognition by the human TSG101 protein. Mol Cell, 2004. 13(6): p. 783-9.

21. Melikova, M.S., K.A. Kondratov, and E.S. Kornilova, Two different stages of epidermal growth factor (EGF) receptor endocytosis are sensitive to free ubiquitin 
depletion produced by proteasome inhibitor MG132. Cell Biol Int, 2006. 30(1): p. 3143.

22. Milhas, D., et al., Anterograde and retrograde transport of neutral sphingomyelinase2 between the Golgi and the plasma membrane. Biochim Biophys Acta, 2010. 1801(12): p. 1361-74.

23. Tomiuk, S., M. Zumbansen, and W. Stoffel, Characterization and subcellular localization of murine and human magnesium-dependent neutral sphingomyelinase. $\mathrm{J}$ Biol Chem, 2000. 275(8): p. 5710-7.

24. Borger, J.G., R. Zamoyska, and D.M. Gakamsky, Proximity of TCR and its CD8 coreceptor controls sensitivity of T cells. Immunol Lett, 2014. 157(1-2): p. 16-22.

25. Yokosuka, T., et al., Spatiotemporal regulation of $T$ cell costimulation by TCR-CD28 microclusters and protein kinase $C$ theta translocation. Immunity, 2008. 29(4): p. 589-601.

26. Vasiliver-Shamis, G., et al., Human immunodeficiency virus type 1 envelope gp120induced partial T-cell receptor signaling creates an F-actin-depleted zone in the virological synapse. J Virol, 2009. 83(21): p. 11341-55.

27. Demetriou, P., et al., A dynamic CD2-rich compartment at the outer edge of the immunological synapse boosts and integrates signals. Nat Immunol, 2020. 21(10): p. 1232-1243.

28. Munoz, P., et al., Antigen-induced clustering of surface CD38 and recruitment of intracellular CD38 to the immunologic synapse. Blood, 2008. 111(7): p. 3653-64.

29. Cockayne, D.A., et al., Mice deficient for the ecto-nicotinamide adenine dinucleotide glycohydrolase CD38 exhibit altered humoral immune responses. Blood, 1998. 92(4): p. 1324-33.

30. Han, L., et al., Phenotypical analysis of ectoenzymes CD39/CD73 and adenosine receptor $2 A$ in CD4(+) CD25(high) Foxp3(+) regulatory T-cells in psoriasis. Australas J Dermatol, 2018. 59(1): p. e31-e38.

31. Samudra, A.N., et al., CD39 and CD73 activity are protective in a mouse model of antiphospholipid antibody-induced miscarriages. J Autoimmun, 2018. 88: p. 131-138.

32. Allard, B., et al., The ectonucleotidases CD39 and CD73: Novel checkpoint inhibitor targets. Immunol Rev, 2017. 276(1): p. 121-144.

33. Clayton, A., et al., Cancer exosomes express CD39 and CD73, which suppress $T$ cells through adenosine production. J Immunol, 2011. 187(2): p. 676-83.

34. Lang, I., et al., Binding Studies of TNF Receptor Superfamily (TNFRSF) Receptors on Intact Cells. J Biol Chem, 2016. 291(10): p. 5022-37.

35. Schubert, D.A., et al., Self-reactive human CD4 $T$ cell clones form unusual immunological synapses. J Exp Med, 2012. 209(2): p. 335-52.

36. Ivey-Hoyle, M., et al., Envelope glycoproteins from biologically diverse isolates of immunodeficiency viruses have widely different affinities for CD4. Proc Natl Acad Sci U S A, 1991. 88(2): p. 512-6.

37. Vasiliver-Shamis, G., et al., Human immunodeficiency virus type 1 envelope gp120 induces a stop signal and virological synapse formation in noninfected CD4+ T cells. J Virol, 2008. 82(19): p. 9445-57.

38. Deng, J., et al., HIV Envelope gp120 Alters T Cell Receptor Mobilization in the Immunological Synapse of Uninfected CD4 T Cells and Augments T Cell Activation. J Virol, 2016.90(23): p. 10513-10526.

39. Schulte, M., et al., ADAM10 regulates FasL cell surface expression and modulates FasL-induced cytotoxicity and activation-induced cell death. Cell Death Differ, 2007. 14(5): p. 1040-9. 
40. Ebsen, H., et al., Subcellular localization and activation of ADAM proteases in the context of FasL shedding in T lymphocytes. Mol Immunol, 2015. 65(2): p. 416-28.

41. Yacoub, D., et al., CD154 is released from T-cells by a disintegrin and metalloproteinase domain-containing protein 10 (ADAM10) and ADAM17 in a CD40 protein-dependent manner. J Biol Chem, 2013. 288(50): p. 36083-93.

42. Bailey, T.L., et al., MEME SUITE: tools for motif discovery and searching. Nucleic Acids Res, 2009. 37(Web Server issue): p. W202-8.

43. Dooley, K., et al., A versatile platform for generating engineered extracellular vesicles with defined therapeutic properties. Mol Ther, 2021. 29(5): p. 1729-1743.

44. Shurtleff, M.J., et al., Y-box protein 1 is required to sort microRNAs into exosomes in cells and in a cell-free reaction. Elife, 2016. 5.

45. Shurtleff, M.J., et al., Broad role for YBX1 in defining the small noncoding RNA composition of exosomes. Proc Natl Acad Sci U S A, 2017. 114(43): p. E8987-E8995.

46. Gay, D., et al., The major histocompatibility complex-restricted antigen receptor on $T$ cells. IX. Role of accessory molecules in recognition of antigen plus isolated IA. J Immunol, 1986. 136(6): p. 2026-32.

47. Biswas, K.H. and J.T. Groves, A Microbead Supported Membrane-Based Fluorescence Imaging Assay Reveals Intermembrane Receptor-Ligand Complex Dimension with Nanometer Precision. Langmuir, 2016. 32(26): p. 6775-80.

48. Gagnon, E., et al., Local changes in lipid environment of TCR microclusters regulate membrane binding by the CD3epsilon cytoplasmic domain. J Exp Med, 2012. 209(13): p. 2423-39.

49. Kim, H.R., et al., T cell microvilli constitute immunological synaptosomes that carry messages to antigen-presenting cells. Nat Commun, 2018. 9(1): p. 3630.

50. Ghosh, S., et al., ERM-Dependent Assembly of T Cell Receptor Signaling and Costimulatory Molecules on Microvilli prior to Activation. Cell Rep, 2020. 30(10): p. 3434-3447 e6.

51. Dong, R., et al., Rewired signaling network in T cells expressing the chimeric antigen receptor (CAR). EMBO J, 2020: p. e104730.

52. Chevillet, J.R., et al., Quantitative and stoichiometric analysis of the microRNA content of exosomes. Proc Natl Acad Sci U S A, 2014. 111(41): p. 14888-93.

53. Bruno Vaz, C.V., Salvatore Valvo, Clara D'Ambra, Francesco Maria Esposito, Valerio Chiurchiù, Oliver Devine, Massimo Sanchez, Giovanna Borsellino, Derek Gilroy, Arne N. Akbar, Michael L. Dustin, Michael Karin, Alessio Lanna, Intercellular telomere transfer extends T cell lifespan. bioRxiv, 2020.

54. Flynn, R.A., et al., Small RNAs are modified with $\mathrm{N}$-glycans and displayed on the surface of living cells. Cell, 2021.

55. Kowal, J., et al., Proteomic comparison defines novel markers to characterize heterogeneous populations of extracellular vesicle subtypes. Proc Natl Acad Sci U S A, 2016. 113(8): p. E968-77.

56. Gould, S.J., A.M. Booth, and J.E. Hildreth, The Trojan exosome hypothesis. Proc Natl Acad Sci U S A, 2003. 100(19): p. 10592-7.

57. Booth, A.M., et al., Exosomes and HIV Gag bud from endosome-like domains of the T cell plasma membrane. J Cell Biol, 2006. 172(6): p. 923-35.

58. Macia, E., et al., Dynasore, a cell-permeable inhibitor of dynamin. Dev Cell, 2006. 10(6): p. 839-50.

59. Uchida, R., et al., Alutenusin, a specific neutral sphingomyelinase inhibitor, produced by Penicillium sp. FO-7436. J Antibiot (Tokyo), 1999. 52(6): p. 572-4. 
60. Bowman, E.J., A. Siebers, and K. Altendorf, Bafilomycins: a class of inhibitors of membrane ATPases from microorganisms, animal cells, and plant cells. Proc Natl Acad Sci U S A, 1988. 85(21): p. 7972-6.

61. Brown, S.S. and J.A. Spudich, Cytochalasin inhibits the rate of elongation of actin filament fragments. J Cell Biol, 1979. 83(3): p. 657-62.

62. Bubb, M.R., et al., Effects of jasplakinolide on the kinetics of actin polymerization. An explanation for certain in vivo observations. J Biol Chem, 2000. 275(7): p. 5163-70.

63. Campi, G., R. Varma, and M.L. Dustin, Actin and agonist MHC-peptide complexdependent T cell receptor microclusters as scaffolds for signaling. J Exp Med, 2005. 202(8): p. 1031-6.

64. Chege, N.W. and S.R. Pfeffer, Compartmentation of the Golgi complex: brefeldin-A distinguishes trans-Golgi cisternae from the trans-Golgi network. J Cell Biol, 1990. 111(3): p. 893-9.

65. Helms, J.B. and J.E. Rothman, Inhibition by brefeldin A of a Golgi membrane enzyme that catalyses exchange of guanine nucleotide bound to ARF. Nature, 1992. 360(6402): p. 352-4.

66. Kucik, D.F., et al., Adhesion-activating phorbol ester increases the mobility of leukocyte integrin LFA-1 in cultured lymphocytes. J Clin Invest, 1996. 97(9): p. 213944.

67. Kupfer, A. and G. Dennert, Reorientation of the microtubule-organizing center and the Golgi apparatus in cloned cytotoxic lymphocytes triggered by binding to lysable target cells. J Immunol, 1984. 133(5): p. 2762-6.

68. Lyubchenko, T.A., G.A. Wurth, and A. Zweifach, The actin cytoskeleton and cytotoxic T lymphocytes: evidence for multiple roles that could affect granule exocytosis-dependent target cell killing. J Physiol, 2003. 547(Pt 3): p. 835-47.

69. Shaikh, S., et al., Bafilomycin-A1 and ML9 Exert Different Lysosomal Actions to Induce Cell Death. Curr Mol Pharmacol, 2019. 12(4): p. 261-271.

70. Teplova, V.V., et al., Bafilomycin Al is a potassium ionophore that impairs mitochondrial functions. J Bioenerg Biomembr, 2007. 39(4): p. 321-9.

71. Tsai, C.C., et al., Dynasore inhibits rapid endocytosis in bovine chromaffin cells. Am J Physiol Cell Physiol, 2009. 297(2): p. C397-406.

72. Tskvitaria-Fuller, I., et al., Regulation of sustained actin dynamics by the TCR and costimulation as a mechanism of receptor localization. J Immunol, 2003. 171(5): p. 2287-95.

73. Young, W.W., Jr., M.L. Allende, and E. Jaskiewicz, Reevaluating the effect of Brefeldin A (BFA) on ganglioside synthesis: the location of GM2 synthase cannot be deduced from the inhibition of GM2 synthesis by BFA. Glycobiology, 1999. 9(7): p. 689-95.

74. Datta, A., et al., Manumycin A suppresses exosome biogenesis and secretion via targeted inhibition of Ras/Raf/ERK1/2 signaling and hnRNP H1 in castrationresistant prostate cancer cells. Cancer Lett, 2017. 408: p. 73-81.

75. Tuladhar, A. and K.S. Rein, Manumycin A Is a Potent Inhibitor of Mammalian Thioredoxin Reductase-1 (TrxR-1). ACS Med Chem Lett, 2018. 9(4): p. 318-322.

76. Pasquier, B., SAR405, a PIK3C3/Vps34 inhibitor that prevents autophagy and synergizes with MTOR inhibition in tumor cells. Autophagy, 2015. 11(4): p. 725-6.

77. Ronan, B., et al., A highly potent and selective Vps34 inhibitor alters vesicle trafficking and autophagy. Nat Chem Biol, 2014. 10(12): p. 1013-9.

78. Pardo, J., et al., Differential implication of protein kinase $C$ isoforms in cytotoxic $T$ lymphocyte degranulation and TCR-induced Fas ligand expression. Int Immunol, 2003. 15(12): p. 1441-50. 
79. Mi, H., et al., PANTHER version 16: a revised family classification, tree-based classification tool, enhancer regions and extensive API. Nucleic Acids Res, 2021. 49(D1): p. D394-D403.

80. Jakka, G., et al., Antigen-specific in vitro expansion of functional redirected NY-ESO1-specific human CD8+ T-cells in a cell-free system. Anticancer Res, 2013. 33(10): p. 4189-201.

81. Maus, M.V., et al., An MHC-restricted antibody-based chimeric antigen receptor requires TCR-like affinity to maintain antigen specificity. Mol Ther Oncolytics, 2016. 3: p. 1-9.

82. Harris, J.R., Negative staining of thinly spread biological samples. Methods Mol Biol, 2007. 369: p. 107-42.

83. Schindelin, J., et al., Fiji: an open-source platform for biological-image analysis. Nat Methods, 2012. 9(7): p. 676-82.

84. Li, D., et al., ADVANCED IMAGING. Extended-resolution structured illumination imaging of endocytic and cytoskeletal dynamics. Science, 2015. 349(6251): p. aab3500.

85. Barbieri, L., et al., Two-dimensional TIRF-SIM-traction force microscopy (2D TIRFSIM-TFM). Nat Commun, 2021. 12(1): p. 2169.

86. Li, D., et al., Astigmatic traction force microscopy (aTFM). Nat Commun, 2021. 12(1): p. 2168.

87. Lamble, S., et al., Improved workflows for high throughput library preparation using the transposome-based Nextera system. BMC Biotechnol, 2013. 13: p. 104.

88. Aparicio-Puerta, E., et al., sRNAbench and sRNAtoolbox 2019: intuitive fast small RNA profiling and differential expression. Nucleic Acids Res, 2019. 47(W1): p. W530-W535.

89. Aparicio-Puerta, E., et al., mirnaQC: a webserver for comparative quality control of miRNA-seq data. Nucleic Acids Res, 2020. 48(W1): p. W262-W267.

90. Chang, L., et al., miRNet 2.0: network-based visual analytics for miRNA functional analysis and systems biology. Nucleic Acids Res, 2020. 48(W1): p. W244-W251.

91. Hale, M.L., I. Thapa, and D. Ghersi, FunSet: an open-source software and web server for performing and displaying Gene Ontology enrichment analysis. BMC Bioinformatics, 2019. 20(1): p. 359.

92. Cox, J., et al., Andromeda: a peptide search engine integrated into the MaxQuant environment. J Proteome Res, 2011. 10(4): p. 1794-805. 
$T$ cell $\rightarrow$ BSLB $\rightarrow$ Conventional
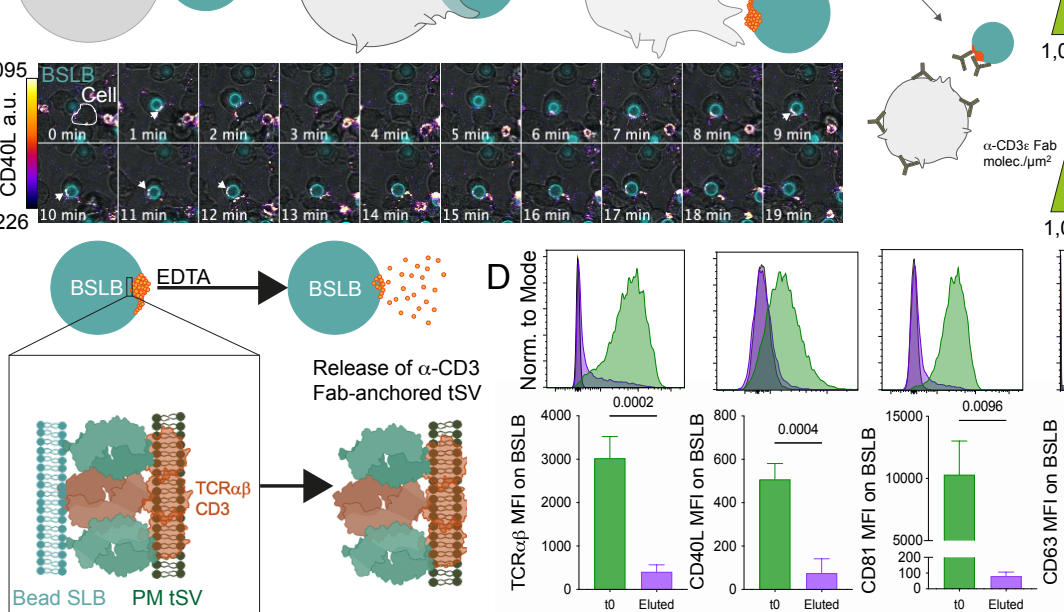

FCM analyses
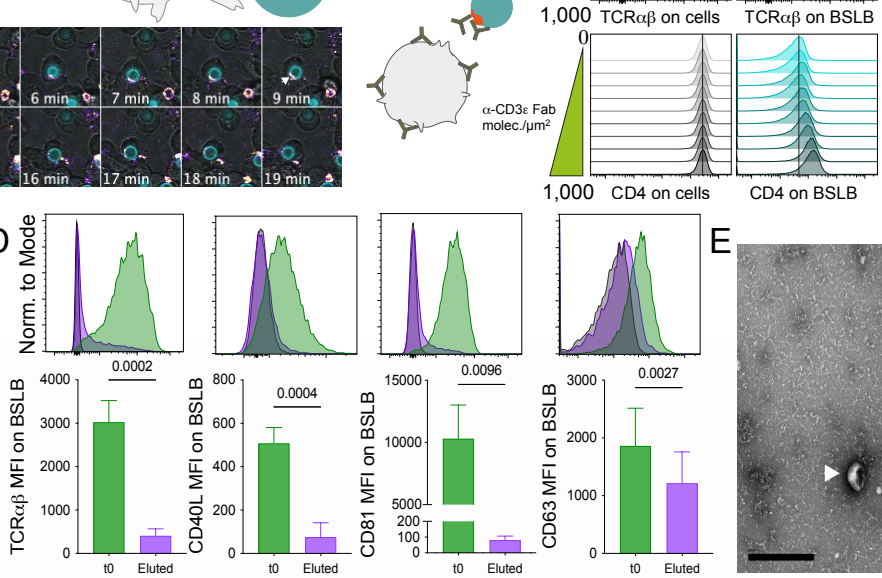

CD2 on cells

D2 on BSLB 1,000 CD4 on cells
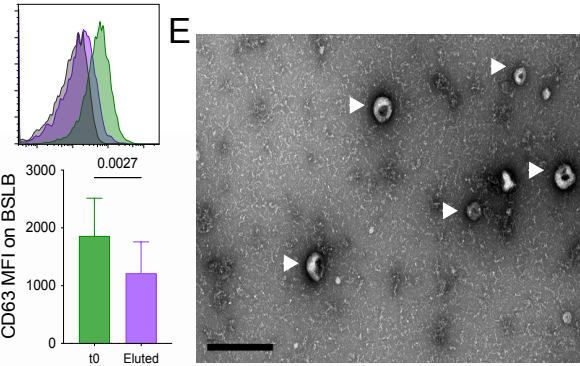

$\mathrm{H}$

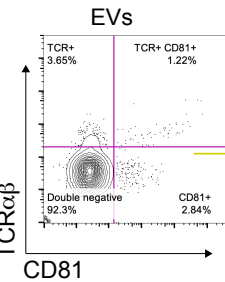
eluate
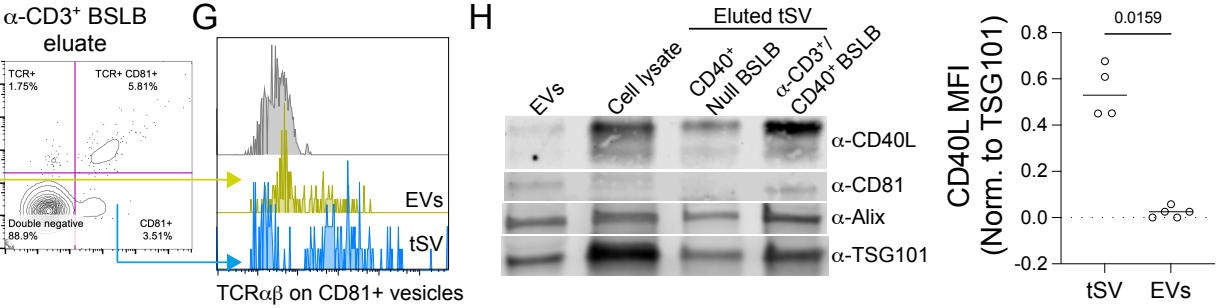

Fig. 1 


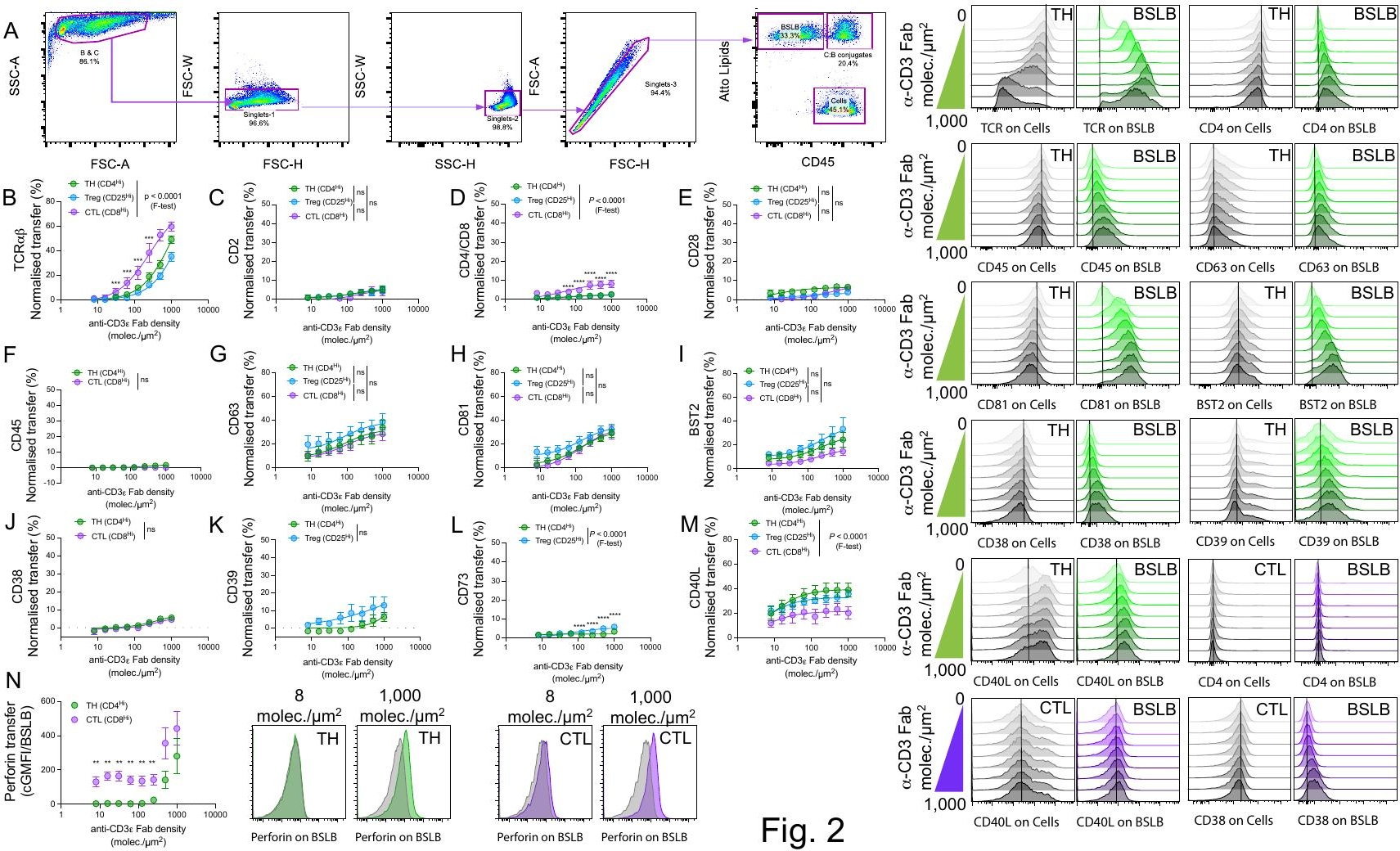


$A$

B

CD40L molec. on BSLB

(Norm. to Ctrl $\mathrm{T}_{\text {max }}$ )

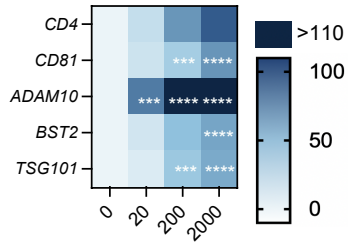

anti-CD3e-Fab (molec./ $\mu \mathrm{m}^{2}$ )

CD81 on BSLB

(Norm. to Ctrl $\mathrm{T}_{\text {max }}$ )

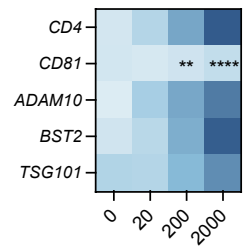

anti-CD3 $\varepsilon$-Fab $\left(\right.$ molec./$\left./ \mu \mathrm{m}^{2}\right)$

$\mathrm{E}$

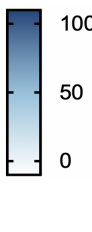

TCR $\alpha \beta$ molec. on BSLB

(Norm. to Ctrl $\mathrm{T}_{\text {max }}$ )
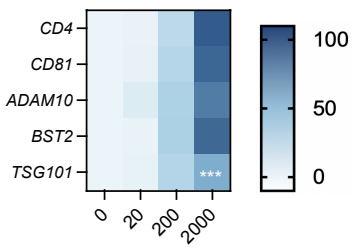

100

50

anti-CD $3 \varepsilon-F a b$

(molec./ $/ \mathrm{m}^{2}$ )

BST2 on BSLB

(Norm. to Ctrl $\mathrm{T}_{\text {max }}$ )

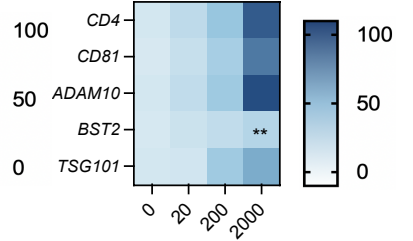

anti-CD3 $\varepsilon-F a b$ (molec./ $\left./ \mathrm{m}^{2}{ }^{2}\right)$

C

CD63 on BSLB

(Norm. to Ctrl $\mathrm{T}_{\max }$ )

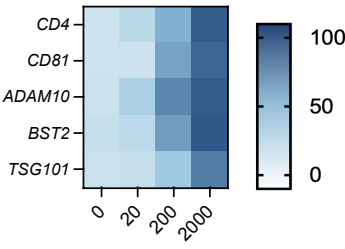

anti-CD3 $\varepsilon-F a b$

(molec./ $\mu \mathrm{m}^{2}$ )

$\mathrm{F}$

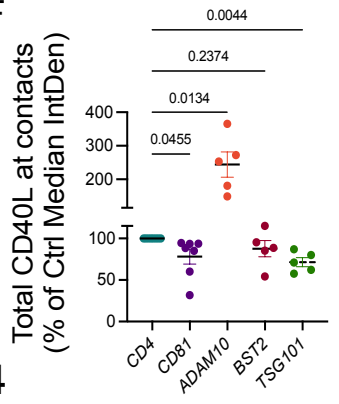
100
$G \quad \alpha$-CD3-Fab IRM ICAM-1 (pseudo) Merge $C D 4$

CD81

ADAM10

BST2

TSG101

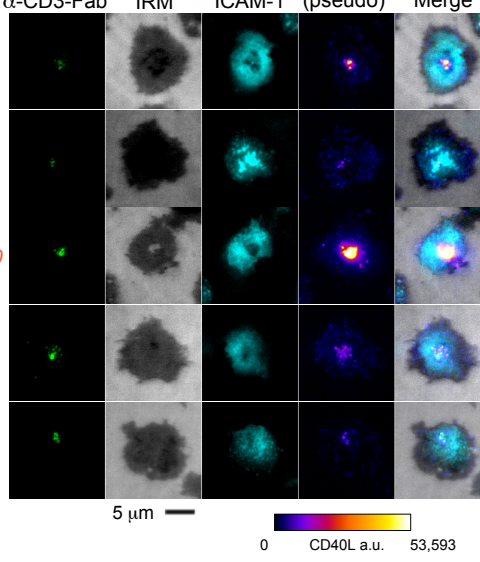

Fig.4

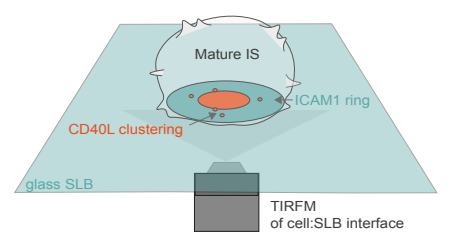


